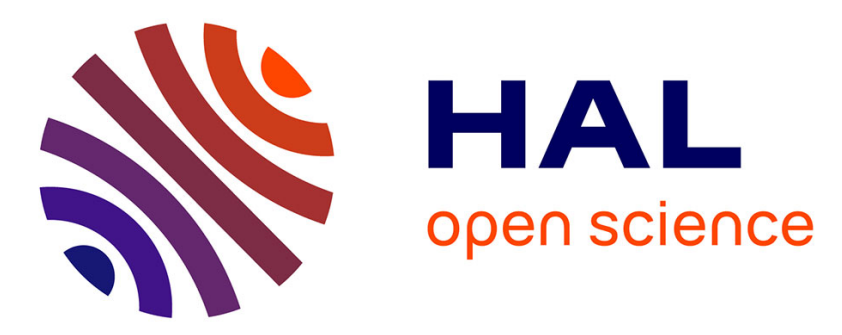

\title{
Restoring glutamate receptosome dynamics at synapses rescues autism-like deficits in Shank3-deficient mice
}

Enora Moutin, Sophie Sakkaki, Vincent Compan, Nathalie Bouquier, Federica

Giona, Julie Areias, Elise Goyet, Anne-Laure Hemonnot-Girard, Vincent

Seube, Bastien Glasson, et al.

\section{To cite this version:}

Enora Moutin, Sophie Sakkaki, Vincent Compan, Nathalie Bouquier, Federica Giona, et al.. Restoring glutamate receptosome dynamics at synapses rescues autism-like deficits in Shank3-deficient mice. Molecular Psychiatry, In press, 10.1038/s41380-021-01230-x . hal-03311533

\section{HAL Id: hal-03311533 \\ https://hal.science/hal-03311533}

Submitted on 2 Aug 2021

HAL is a multi-disciplinary open access archive for the deposit and dissemination of scientific research documents, whether they are published or not. The documents may come from teaching and research institutions in France or abroad, or from public or private research centers.
L'archive ouverte pluridisciplinaire HAL, est destinée au dépôt et à la diffusion de documents scientifiques de niveau recherche, publiés ou non, émanant des établissements d'enseignement et de recherche français ou étrangers, des laboratoires publics ou privés. 


\title{
Restoring glutamate receptosome dynamics at synapses rescues autism-like deficits in Shank3-deficient mice
}

\author{
Enora Moutin $1^{凶}$, Sophie Sakkaki ${ }^{1,6}$, Vincent Compan ${ }^{1,6}$, Nathalie Bouquier ${ }^{1,6}$, Federica Giona ${ }^{2}$, Julie Areias ${ }^{1}$, Elise Goyet ${ }^{3}$, \\ Anne-Laure Hemonnot-Girard 1, Vincent Seube ${ }^{1}$, Bastien Glasson ${ }^{1}$, Nathan Benac ${ }^{3}$, Yan Chastagnier 1, Fabrice Raynaud ${ }^{1,4}$, \\ Etienne Audinat ${ }^{1}$, Laurent Groc ${ }^{3}$, Tangui Maurice ${ }^{5}$, Carlo Sala ${ }^{2}$, Chiara Verpelli ${ }^{2}$ and Julie Perroy
}

Shank3 monogenic mutations lead to autism spectrum disorders (ASD). Shank3 is part of the glutamate receptosome that physically links ionotropic NMDA receptors to metabotropic mGlu5 receptors through interactions with scaffolding proteins PSD95-GKAP-Shank3-Homer. A main physiological function of the glutamate receptosome is to control NMDA synaptic function that is required for plasticity induction. Intact glutamate receptosome supports glutamate receptors activation and plasticity induction, while glutamate receptosome disruption blocks receptors activity, preventing the induction of subsequent plasticity. Despite possible impact on metaplasticity and cognitive behaviors, scaffold interaction dynamics and their consequences are poorly defined. Here, we used mGlu5-Homer interaction as a biosensor of glutamate receptosome integrity to report changes in synapse availability for plasticity induction. Combining BRET imaging and electrophysiology, we show that a transient neuronal depolarization inducing NMDA-dependent plasticity disrupts glutamate receptosome in a long-lasting manner at synapses and activates signaling pathways required for the expression of the initiated neuronal plasticity, such as ERK and mTOR pathways. Glutamate receptosome disruption also decreases the NMDA/AMPA ratio, freezing the sensitivity of the synapse to subsequent changes of neuronal activity. These data show the importance of a fine-tuning of protein-protein interactions within glutamate receptosome, driven by changes of neuronal activity, to control plasticity. In a mouse model of ASD, a truncated mutant form of Shank3 prevents the integrity of the glutamate receptosome. These mice display altered plasticity, anxiety-like, and stereotyped behaviors. Interestingly, repairing the integrity of glutamate receptosome and its sensitivity to the neuronal activity rescued synaptic transmission, plasticity, and some behavioral traits of Shank $3 \Delta \mathrm{C}$ mice. Altogether, our findings characterize mechanisms by which Shank3 mutations cause ASD and highlight scaffold dynamics as new therapeutic target.

\section{INTRODUCTION}

Autism spectrum disorders (ASD) constitute a group of neurodevelopmental disorders characterized by deficits in social communication and stereotyped behaviors such as restricted and repetitive behaviors and interests or activities (DSM5, https://doi. org/10.1176/appi.books.9780890425596). At least 25\% of ASD have a genetic etiology and many of the associated genes involved regulate synaptic plasticity, leading to the "synaptic hypothesis." According to this hypothesis, ASD would be the consequence of synaptic impairment [1]. Mutations in the SHANK3 gene are one of the most common genetic causes of ASD, together termed "Shankopathies" [2-5]. Many mouse models of Shankopathies exist and recapitulate cognitive deficits found in patients, making them relevant to study the synaptic hypothesis. Shank3-deficient mice exhibit molecular impairments causing deficits in NMDA receptor-mediated neurotransmission associated with cognitive deficits [6-10] but little is known about how mutations in SHANK3 contribute to these dysfunctions. To tackle this issue, we used Shank $3 \Delta \mathrm{C}$ mice. These mice have a disruption of Shank3 gene at exon 21. This haploinsufficiency of Shank3 gene causes mechanism of ASD in humans [11] and also triggered related phenotypes in Shank3 $\Delta C$ mice [7]. Here, we explored the molecular dynamics of Shank3 complex at synapses following neuronal excitation in WT and Shank $3 \Delta \mathrm{C}$ mice and its relevance for the control of glutamate receptors function, plasticity, and behavior. In addition, we devised a strategy to rescue synaptic functioning in Shank $3 \Delta C$ mice.

The postsynaptic density (PSD) of excitatory synapses is complex and dynamic in composition and regulation [12]. Shank3 appears as the centerpiece of the PSD. This scaffolding protein contains an N-terminal ankyrin repeat domain, a SH3 domain, a PDZ domain, a proline-rich domain, and a C-terminal SAM domain $[13,14]$. By linking together major actors of the PSD, Shank3 is deeply involved in the organization and function of the glutamatergic post-synapse [15-17]. It physically interacts with the C-terminal domain of GKAP through its PDZ domain and with Homer EVH1 (Ena/Nasp homology) N-terminal domain through its proline-rich domain, thereby creating a physical molecular bridge

${ }^{1}$ IGF, University of Montpellier, CNRS, INSERM, Montpellier, France. ${ }^{2}$ Cnr Neuroscience Institute, 3220129 Milan, Italy. ${ }^{3}$ Interdisciplinary Institute for NeuroScience, CNRS, UMR 5297, Centre Broca Nouvelle-Aquitaine, 33076 Bordeaux, France. ${ }^{4}$ PhyMedExp, Univ Montpellier, INSERM, CNRS, CHU de Montpellier, France. ${ }^{5}$ MMDN, Univ Montpellier, EPHE, INSERM, Montpellier, France. ${ }^{6}$ These authors contributed equally: Sophie Sakkaki, Vincent Compan, Nathalie Bouquier. ${ }^{凶}$ email: enora.moutin@igf.cnrs.fr; julie.perroy@igf.cnrs.fr 


\section{Activatable NMDA receptors

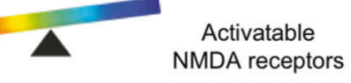 \\ NMDA receptors}
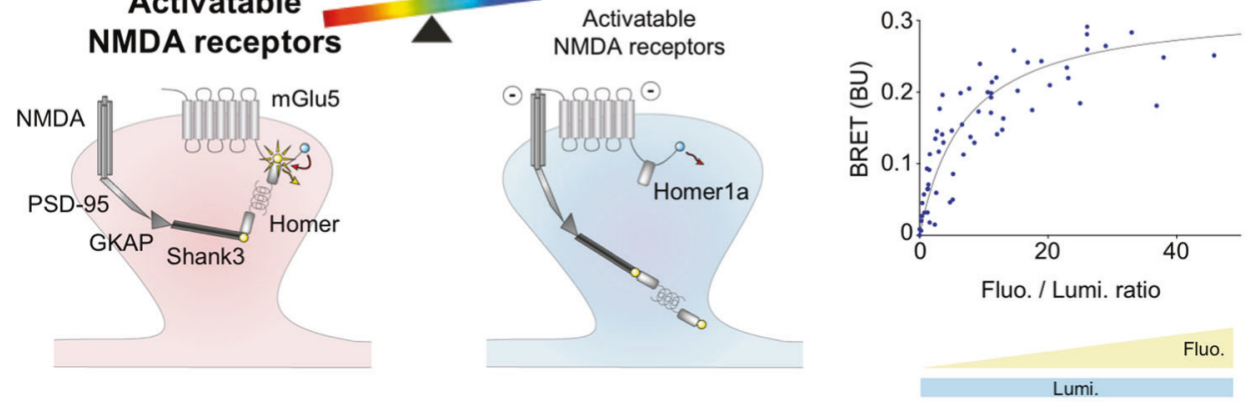

C

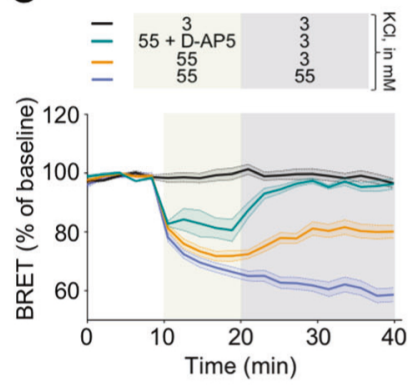

D

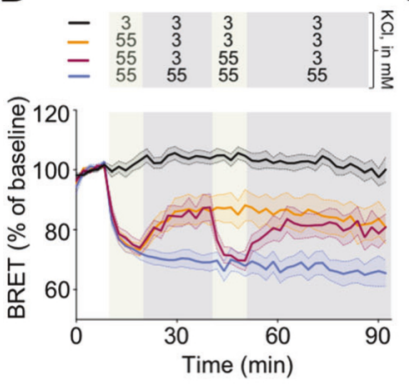

$\mathbf{E}$
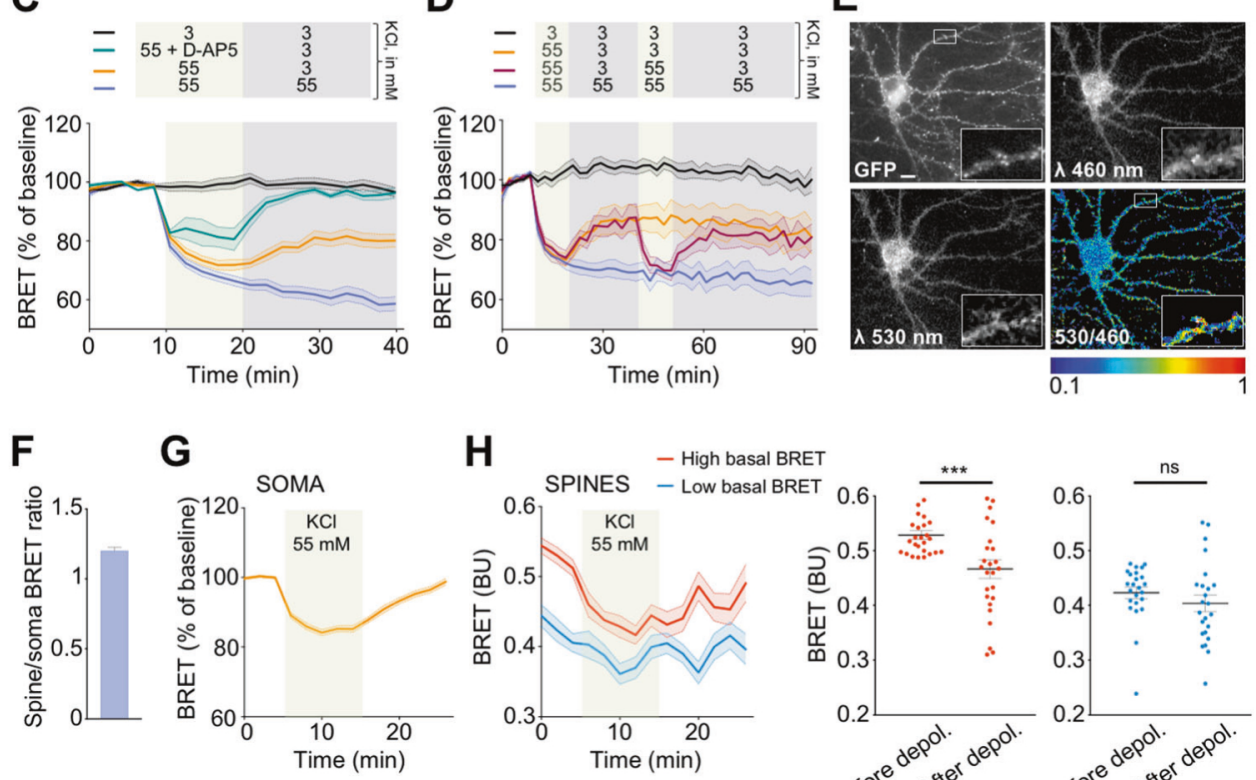

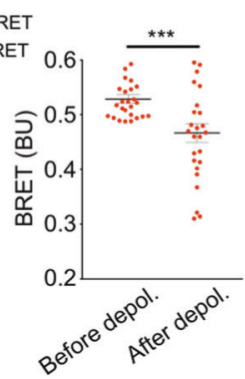

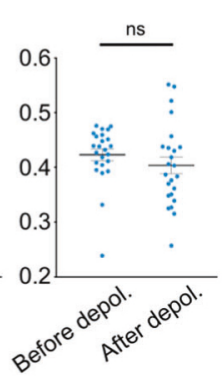

Fig. 1 Transient depolarization induces a long-lasting disruption of mGlu5-Homer interaction in spines. A Schematic illustrations of the glutamate receptosome organization in spines from WT mice. BRET intensities between mGlu5-NLuc and Venus-Homer enables to report intact (left) or disrupted (right) complexes, which are respectively highlighting synapses that are available or not for plasticity induction. BRET recordings between mGlu5-NLuc and Venus-Homer in neurons from hippocampal cultures in cell population using a fluorometer (B-D) or in microscopy (E-H). BU stands for BRET units. B Individual readings obtained from five independent experiments were pooled. Curves were fitted using a nonlinear regression equation, assuming a single binding site (GraphPad Prism version 7). C, D, G, H Real-time measurements of BRET variations measured in neurons during changes of membrane potential with $\mathrm{KCl} 3$ and $55 \mathrm{mM}$. Data are mean \pm SEM of BRET intensities recorded in triplicate from at least three independent experiments (C, D) or on 18-45 neurons in at least six independent experiments $(\mathbf{G}, \mathbf{H})$. In C, green circles, D-AP5 $100 \mu \mathrm{M}$ was added during the transient depolarization. E Example of BRET imaging recorded by videomicroscopy in one neuron. The pictures show expression of Venus-Homer1c (GFP), mGlu5-NLuc (Em $460 \mathrm{~nm}$ ), Venus-Homer1c excited by the energy transfer coming from mGlu5-NLuc (Em $530 \mathrm{~nm}$ ), and the BRET signal (530/460 ratio). Scale bar $10 \mu \mathrm{m}$. F Ratio of BRET intensities recorded in spines over soma on 45 neurons from eight independent cultures. H Left panel, BRET variations recorded on 50 dendritic spines segregated according to the intensity of their basal BRET: higher (red), or lower (blue) than 0.48 , the median BRET value measured on the three first time points. Right panels, BRET intensities in spines measured on the three first (before depol.) or three last (after depol.) time points in spines with high (red) or low (blue) basal BRET. ns, ${ }^{* * *}$ indicate $p$ value $>0.05,<0.001$, respectively; Paired $t$ test. Point-by-point statistical tests and post hoc comparisons for $\mathbf{C}, \mathbf{D}, \mathbf{G}$, and $\mathbf{H}$ (left) are indicated in Supplementary Table S1.

between ionotropic NMDA and metabotropic mGlu5 receptors through the PSD95-GKAP-Shank-Homer complex, which we call the "glutamate receptosome" (Fig. 1A). These receptor-associated scaffolding proteins are much more than just placeholders since they can modulate receptors expression and activity, and therefore modulate their associated signaling pathways (see $[18,19]$ for reviews). The synaptic protein interaction network undergoes input-dependent rearrangement by neuronal activity [20]. In this context, the dynamics of the scaffolding complex play the leading role in the control of receptor functioning and synaptic transmission. Because mGlu5 and NMDA receptors play tremendous roles in the induction of synaptic plasticity, fine regulations of scaffold interactions within the glutamate receptosome finely tune the availability of synapses for plasticity induction [21]. A noteworthy example of these molecular dynamics is the one controlled by Homer isoforms [22]. The constitutive long forms of Homer ensure the glutamate receptosome integrity by linking Shank3 to mGlu5 receptor [23] through the multimerization of Homer [24]. In contrast, a short monomeric form of Homer, Homer1a, encoded by an immediate-early gene (IEG) after a sustained neuronal activity, competes with the multimeric form of Homer to interact with mGlu5 receptor, freeing it from the complex [22]. When the scaffolding complex is disrupted, mGlu5 and NMDA receptors can directly interact, resulting in a reciprocal functional inhibition 
$[21,25]$. This restructuring occurs after sustained neuronal activity (like those required to induce long-term potentiation) and serves as negative feedback to decrease synaptic NMDA receptor activity. As a consequence, the efficacy of synaptic transmission is locked, preventing further induction of synaptic plasticity (Fig. 1A). However, very little is known about the properties of these molecular dynamics at synapses to promote metaplasticity. The question of how these scaffolds remodel over time, in response to neuronal activity, remains open. A better understanding of this molecular tinkering would enable to repair deficient interactions in autism models.

Here, we used mGlu5-Homer interaction as a biosensor of the glutamate receptosome integrity in WT mice to report the spatiotemporal changes of synaptic NMDA functioning, in living cells. We found that a NMDA-dependent transient increase in neuronal activity disrupts mGlu5-Homer interaction in a long-lasting manner at synapses, inducing signaling involved in plasticity such as ERK and mTOR pathway activation. Scaffold disruption also triggers feedback inhibition of NMDA/AMPA current ratio. Hence, disrupting mGlu5-Homer interaction before plasticity induction abolished ERK/mTOR activity-dependent signaling, highlighting the role of scaffold remodeling in metaplasticity. These last conditions were similar to those that apply to Shank3 $\Delta C$ mice: the glutamate receptosome is atypically disrupted by the lack of interaction between the truncated form of Shank3 and Homer, furthermore NMDA/AMPA current ratio is decreased compared to WT mice. However, by expressing a molecular bridge that restores glutamate receptosome integrity in Shank $3 \Delta C$ mice and its sensitivity to neuronal activity, we could recover the NMDA/AMPA ratio, rescue ERK and mTOR signaling, and improve autistic-like behaviors such as anxiety and repetitive stereotyped behavior. Modulation of glutamate receptosome integrity could therefore represent a new therapeutic strategy in Shankopathies and more largely in ASD.

\section{MATERIALS AND METHODS}

\section{Animal handling}

All animal procedures were conducted in accordance with the European Communities Council Directive, supervised by the IGF Institute's Local Animal Welfare Unit (A34-172-41) and approved by the French Ministry of Research (agreement numbers: APAFIS\#23357-2019112715218160 v4 and APAFIS\#23476-2020010613546503 v4). The Shank3 $\Delta C$ mice have a deletion of exon 21 that includes the Homer-binding domain (Jackson laboratory, Bar Harbor, ME) and were backcrossed to C57BL6/J mice. Heterozygous mice were bred and all mice were grouped after weaning with respect to their sex. For experiments, we used homozygous Shank3 $\Delta C$ mice and WT littermates.

\section{Solutions used for experiments}

$\mathrm{KCl} 3 \mathrm{mM}$ (in mM): $\mathrm{NaCl} 140, \mathrm{CaCl}_{2} 2, \mathrm{KCl} 3$, Hepes 10, D-glucose 10, glycine 0.01 , bicuculline $0.01,0.0003$ tetrodotoxin, $\mathrm{pH} 7.4$ with an osmolarity around $315-320 \mathrm{mOsm}$.

Membrane potential was calculated using the voltage Goldman-Hodgkin-Katz (GHK) equation. This equation takes into consideration two main factors: (1) the membrane permeability and (2) concentration gradient of the most abundant permeable ions, $\mathrm{Na}^{+}, \mathrm{K}^{+}$, and $\mathrm{Cl}^{-}$.

$V_{\mathrm{m}}=\frac{R T}{F} \ln \left(\frac{p_{\mathrm{k}}\left[\mathrm{K}^{+}\right]_{\mathrm{o}}+p_{\mathrm{Na}}\left[\mathrm{Na}^{+}\right]_{\mathrm{o}}+p_{\mathrm{Cl}}\left[\mathrm{CL}^{-}\right]_{\mathrm{i}}}{p_{\mathrm{k}}\left[\mathrm{K}^{+}\right]_{\mathrm{i}}+p_{\mathrm{Na}}\left[\mathrm{Na}^{+}\right]_{\mathrm{i}}+p_{\mathrm{Cl}}\left[\mathrm{CL}^{-}\right]_{\mathrm{o}}}\right)$

$V_{\mathrm{m}}=$ membrane potential.

$R=$ universal gas constant $\left(8.314 \mathrm{~J} \cdot \mathrm{K}^{-1} \cdot \mathrm{mol}^{-1}\right)$.

$T=$ temperature in Kelvin $\left(\mathrm{K}={ }^{\circ} \mathrm{C}+273.15\right)$.

$F=$ Faraday's constant $\left(96485 \mathrm{C} \cdot \mathrm{mol}^{-1}\right)$.

$p_{\mathrm{K}}=$ membrane permeability for $\mathrm{K}^{+} \cdot p_{\mathrm{K}}: p_{\mathrm{Na}}: p_{\mathrm{Cl}}=1: 0.05: 0.45$.

$\left[\mathrm{K}^{+}\right]_{\mathrm{O}}$ is the concentration of $\mathrm{K}^{+}$in the extracellular solution.

$\left[\mathrm{K}^{+}\right]_{i}$ is the concentration of $\mathrm{K}^{+}$in the intracellular solution.

According to $\mathrm{GHK}$ equation, neurons in $\mathrm{KCl} 3 \mathrm{mM}$ present a membrane potential of $-71 \mathrm{mV}$.
$\mathrm{KCl} 55 \mathrm{mM}$-evoked depolarization solution (in $\mathrm{mM}$ ): $\mathrm{NaCl} 88, \mathrm{CaCl}_{2} 2, \mathrm{KCl}$ 55, Hepes 10, D-glucose 10, glycine 0.01 , bicuculline $0.01,0.0003$ tetrodotoxin, $\mathrm{pH} 7.4$ with an osmolarity around 315-320 mOsm. According to $\mathrm{GHK}$ equation, this $\mathrm{KCl} 55 \mathrm{mM}$-containing solution raises membrane potential to $-30 \mathrm{mV}$.

\section{Plasmids, BRET biosensors, and TAT peptides}

Packaging plasmid pMD2.G and psPAX2 plasmids were given by Didier Trono (Addgene plasmid \#12259 and \#12260).

For transgene expression, backbones of pWPT-Camk2aprom-mGlu5NLuc and pWPT-Camk2aprom-Venus-Homer1C were all derived from pWPT-GFP plasmid (Addgene plasmid \#12255). pAAV-CW3SL-mGlu5-NLuc was derived from pAAV-CW3SL-eGFP (given by Bong-Kiun Kaang, Addgene plasmid \#61463). All these plasmids were produced by Gibson Assembly (New England Biolabs) after amplification by PCR of mGlu5, Venus, Homer1C, or CamK2a promoter. pAAV-CW3SL-Venus-Homer1b-GluN2BCtail was obtained by subcloning the Venus sequence from pAAV-CW3SLVenus-Homer1C and the Homer1b-GluN2B-Ctail sequence from pGW1-GFP-Homer1b-GluN2B-Ctail into pAAV-CW3SL-mGlu5-NLuc using Gibson Assembly Protocol. pGW1-GFP-Homer1b-NR2B-Ctail was built by adding the last eight AA of GluN2B (LSSIESDV) spaced by 10 glycine to the C-terminal of Homer $1 b$ protein.

YEN BRET biosensor, which reports ERK activity, was already described [26] and subcloned in a pAAV backbone to obtain pAAV-hSyn-YEN. AIMTOR BRET biosensor, which reports mTORC1 activity, was already described [27] (pAAV-hSyn-T757-AIMTOR).

Cell-permeable TAT-fused peptides TAT C-tail (YGRKKRRQRRRALTPPSPFR) and the control TAT (TAT ctrl: YGRKKRRQRRR-ALTPLSPRR) [28] were synthesized by Smart Bioscience company, resuspended in $\mathrm{H}_{2} \mathrm{O}$ at a concentration of $1 \mathrm{mM}$, and stored at $-80^{\circ} \mathrm{C}$. Please note that TAT Ctail can disrupt mGlu5-Homer as well as Shank3-Homer interaction as it mimics the proline-rich EVH1 binding domain that is present in mGlu5 and Shank3. TATs were used at $5 \mu \mathrm{M}$ for BRET experiments and at $10 \mu \mathrm{M}$ for electrophysiology.

\section{HEK293T culture}

HEK293T cells were cultured in DMEM (Invitrogen) containing 10\% FBS and antibiotics (penicillin/streptomycin).

\section{Viral productions}

Lentiviral particles were produced as described [29]. Briefly, HEK293T cells were cotransfected with the targeted vector plasmid, the packaging plasmid pMD2G, and the envelope plasmid pSPAX2 using phosphate calcium technique. After 3 days of incubation, the cell supernatant was filtered and incubated overnight with polyethylene glycol to allow lentivirus concentration by precipitation. After centrifugation, pellets containing lentiviral particles were resuspended in PBS, aliquoted, and stored at $-80^{\circ} \mathrm{C}$.

AAV-DJ particles were produced using a commercial kit, according to the manufacturer's protocol (AAV purification kit, reference 6666 from Takara and reference VPK-140 from Cell Biolabs). AAV particles were resuspended in PBS with an approximate titer of $1 \times 10^{14}$ genome copies/ $\mathrm{ml}$, aliquoted and stored at $-80^{\circ} \mathrm{C}$.

\section{Hippocampal primary cell culture and transduction}

For the majority of the experiments, hippocampal neuronal primary cultures were prepared from P0-P2 mice as described [29]. Briefly, brain hippocampi were mechanically and enzymatically dissociated with papain (Sigma-Aldrich) and hippocampal cells were seeded in Neurobasal-A medium (Gibco) supplemented with B-27 (Gibco), Glutamax (Gibco), Lglutamine (Gibco), antibiotics (Gibco), and fetal bovine serum (Gibco). After 2 days in culture, cytosine $\beta$-D-arabinofuranoside hydrochloride (SigmaAldrich) was added to curb glia proliferation. The day after, $75 \%$ of the medium was replaced by BrainPhys medium (Stemcell Technologies) supplemented with B-27 (Gibco), Glutamax (Gibco), and antibiotics (Gibco). At DIV6, $15 \%$ of the medium was replaced by fresh medium containing lentiviral particles or AAV particles for neuronal transduction, as recently described [29]. For immunocytochemistry and for single particle tracking experiments hippocampal neuronal primary cultures were prepared from embryonic rats (E18). Briefly, brain hippocampi were mechanically and enzymatically dissociated with trypsin (Sigma-Aldrich) and hippocampal cells were seeded in neurobasal medium (Gibco) supplemented as for postnatal cultures. After 3 days in culture, cytosine $\beta$-D-arabinofuranoside hydrochloride (Sigma-Aldrich) was added only for cultures from mice. 


\section{Coimmunoprecipitation}

Hippocampi from 14 Shank $3 \Delta C$ mice and 14 WT littermates (2-4 months old) were solubilized in cold lysis buffer (in $\mathrm{mM}$ ): Tris- $\mathrm{HCl}$ (50), $\mathrm{NaCl}(120)$, $\mathrm{NaF}(50)$, and Triton $1 \%$ supplemented with protease and phosphatase inhibitors (Thermo Fisher). After 10 up and down using Dounce homogenizer, samples were kept on ice for $30 \mathrm{~min}$ and centrifuged at $15.900 \times g$ for $10 \mathrm{~min}$ at $4{ }^{\circ} \mathrm{C}$. Concentration of solubilized proteins was quantified using BCA assay (Sigma-Aldrich). All samples were then set at the same volume and concentration for the rest of the protocol. One equal volume was kept for total extracts and the rest was incubated with antibody against mGlu5 (rabbit clone D6E7B from Cell Signaling) with rotation overnight at $4{ }^{\circ} \mathrm{C}$. Then, protein A/G magnetic beads (Pierce, Life technologies) were added in samples for $4 \mathrm{~h}$ with rotation at $4{ }^{\circ} \mathrm{C}$. After washings, proteins were eluted in LDS sample buffer (Thermo Fisher) supplemented with $2 \%$ ß-mercaptoethanol, resolved on $4-12 \%$ gradient NuPAGE Bis-Tris gels (Thermo Fisher), transferred onto nitrocellulose membranes, and detected by immunoblot using the following primary antibodies: mGlu5 (rabbit clone D6E7B from Cell Signaling, dilution 1:1000), actin (mouse clone from DSHB, reference JLA20, dilution 1:2000), and GluN1 (rabbit clone D65B7 from Cell Signaling, dilution 1:1000).

\section{BRET measurements}

Just before BRET recordings, the culture medium was replaced by a $\mathrm{KCl} 3$ $\mathrm{mM}$ solution. To assess the effects of depolarization on BRET signal, $\mathrm{KCl} 3$ $\mathrm{mM}$ was replaced by a $\mathrm{KCl} 55 \mathrm{mM}$ solution. $\mathrm{KCl} 3$ and $55 \mathrm{mM}$ solutions are described above.

Cell-population BRET measurements were performed using the Infinite F500 (TECAN) 96-well plate reader on DIV 14-16 neurons previously transduced at DIV6 with pWPT-Camk2aprom-mGlu5-NLuc and pWPTCamk2aprom-Venus-Homer1C or with pAAV-CW3SL-mGlu5-NLuc and pAAV-CW3SL-Venus-Homer1b-GluN2B-Ctail. BRET computed as the ratio of the intensity detected from the Venus acceptor entity $(520-575 \mathrm{~nm}$ band-pass filter, Em $530 \mathrm{~nm}$ ) to the nano-luciferase (NLuc) donor (390-450 $\mathrm{nm}$ band-pass filter, Em $460 \mathrm{~nm}$ ), after the addition of $20 \mu \mathrm{M}$ furimazine. BRET saturation was measured in neurons transduced with constant concentrations of mGlu5-NLuc and increasing concentrations of Venus-Homer1c. BRET was measured in each well every 2 min (three wells per condition in each experiment, pooled for the analysis).

Single-cell BRET imaging was performed as previously described [26, 30] on hippocampal neurons from Shank $3 \Delta C$ or WT mice. Neurons cultured on glass-bottom culture dishes (reference 81156-400, Ibidi) were transduced at DIV6 with pWPT-Camk2aprom-mGlu5-NLuc and pWPT-Camk2apromVenus-Homer1C or with pAAV-hSyn-T757-AIMTOR or with pAAV-hSyn-YEN and/or with pCW3SL-Venus-Homer1b-GluN2B-Ctail. We performed acquisitions between DIV13 and DIV15 for experiments on ERK and mTOR and between DIV15 and DIV17 for experiments on mGlu5-Homer1 interaction. All images were obtained using a bioluminescence-dedicated inverted fluorescence microscope (Axiovert $200 \mathrm{M}$, Carl Zeiss) with a plan apochromat $63 \times / 1.40$ oil M27 objective at room temperature and collected with an Evolve camera (Photometrics) equipped with an EMCCD detector back-illuminated, on-chip multiplication gain. Sequential acquisitions of light emission at 460 and $530 \mathrm{~nm}$ were performed at $5 \mathrm{MHz}$, Em gain 200 and conversion gain $3 e^{-} / A D U$, binning 1 , with emission filters FF01-450/ 70-25 (Semrock) and HQ535/50 nm (No. 63944, Chroma), respectively. Furimazine $(50 \mu \mathrm{M})$ was applied $1 \mathrm{~min}$ before acquisition and the $530 \mathrm{~nm} /$ $460 \mathrm{~nm}$ ratio was read every $2 \mathrm{~min}$ for each neuron. For BRET between mGlu5 and Homer and for AIMTOR biosensor, we sequentially exposed the donor $10 \mathrm{~s}$ and the acceptor $20 \mathrm{~s}$. For ERK biosensor, we sequentially exposed the donor $5 \mathrm{~s}$ and the acceptor $10 \mathrm{~s}$. BRET was measured in each field every 2 or $3 \mathrm{~min}$. Analysis was performed as previously described [31]. Briefly, we used an open source toolset for Fiji (https://github.com/ ychastagnier/BRET-Analyzer) that performs four key steps: (1) image background subtraction, (2) image alignment over time, (3) a composite thresholding method of the image, and (4) pixel by pixel division of the image and distribution of the ratio intensity on a pseudocolor scale.

\section{AAV injections and immunohistochemistry}

We injected Shank $3 \Delta C$ and WT male mice littermates between 6 and 8 postnatal days. Mice were anesthetized with isoflurane (Vetflurane ${ }^{\circ}, 2-5 \%$ ) and $A A V$ vectors were injected into the lateral ventricles randomly across the different litters. $0.5 \mu \mathrm{l}$ were injected through each injection site at the following coordinates respective to bregma: antero-posterior: $-2.5 \mathrm{~mm}$, median-lateral: $-2.5 \mathrm{~mm} /+2.5 \mathrm{~mm}$, dorso-ventral: $-1.25 \mathrm{~mm}$ based on Allen Developing Mouse Brain Atlas (2008). After weaning, mice were divided in three groups and kept in separate cages (six cages per group), WT injected with AAV containing eGFP, Shank $3 \Delta C$ injected with AAV containing eGFP, and Shank3 $\triangle C$ injected with AAV containing Homer-GluN2B chimaera. Wald Chi-square test performed on generalized estimated equation estimated marginal means, with group and cage as factors and each specific behavioral measurement as variable, ruled out potential cage effect on grooming $(p=0.763)$, total distance $(p=0.240)$, and speed $(p=0.155)$ in the open field. For the percentage of time spent in the center of the open field, the marble burying and the fear-conditioning Wald Chi-square test was indicating a $p$ value $<0.001$ for a cage effect. This was due to the effect of three cages coming from the same batch of animals ( $n=5$ in each group) and was true for the three experimental groups within this batch. This batch effect does not influence overall the differences seen between our experimental groups. To evaluate the efficiency of the viral infection, mice brains were kept after behavioral experiments in $4 \%$ paraformaldehyde during $24 \mathrm{~h}$, washed, and then sliced into coronal sections of $30 \mu \mathrm{m}$ thickness using a vibratome. Sections were processed for immunofluorescence to enhance eGFP signal. Briefly, slices were permeabilized in PBS containing Triton $0.2 \%$, then blocked with PBS containing $\mathrm{BSA} 3 \%$, and incubated overnight with an antibody against eGFP (chicken, AVES, GFP-1020) diluted in PBS containing BSA $1 \%$ and Triton $0.1 \%$ and then incubated with appropriate secondary antibody. Images were acquired using an Axiolmager Z1 Zeiss microscope equipped for optical sectioning (Apotome) and with appropriate epifluorescence filters. Supplementary Fig. S8 shows transgene expression in brain areas.

\section{Electrophysiology in acute slices}

Shank $3 \Delta C$ mice or WT littermates aged between postnatal days 14 and 20 were briefly anesthetized with isoflurane and decapitated. Brains were quickly removed in oxygenated $\left(5 \% \mathrm{CO}_{2}\right.$ and $\left.95 \% \mathrm{O}_{2}\right)$ ice-cold extracellular solution containing (in $\mathrm{mM}$ ): sucrose (215), D-glucose (20), sodium pyruvate (5), $\mathrm{KCl}(2.5), \mathrm{NaH}_{2} \mathrm{PO}_{4}$ (1.25), $\mathrm{NaHCO}_{3}$ (25.9), $\mathrm{MgCl}_{2}$ (7), and $\mathrm{CaCl}_{2}$ (1) $(\mathrm{pH} 7.4,315 \mathrm{mOsm})$. Acute coronal slices $350 \mu \mathrm{m}$ thick were cut using a Leica VT1200S vibratome in the same extracellular solution and then incubated at $33^{\circ} \mathrm{C}$ in ACSF (in mM): $\mathrm{NaCl}$ (126), D-glucose (20), sodium pyruvate (5), $\mathrm{KCl}$ (2.5), $\mathrm{NaH}_{2} \mathrm{PO}_{4}$ (1.25), $\mathrm{NaHCO}_{3}$ (25.9), $\mathrm{MgCl}_{2}$ (1), and $\mathrm{CaCl}_{2}$ (2) $(\mathrm{pH} 7.4,310 \mathrm{mOsm})$. Extracellular solution temperature was then slowly cooled until reaching room temperature and slices were maintained in this oxygenated solution until recordings $(0.5-5 \mathrm{~h})$. Before recordings, a cut was made between CA3 and CA1 to prevent the propagation to CA1 of CA3generated bursts of action potentials upon blockade of GABA-A receptors. For recordings, slices were transferred in a recording chamber, perfused with ACSF $(2-3 \mathrm{ml} / \mathrm{min})$ at room temperature. CA1 pyramidal cells were visually selected using infrared LED illumination (Scientifica Ltd.) and an Olympus 40x water immersion objective. For experiments on mice injected with viruses, fluorescent CA1 pyramidal neurons were selected using a $480 \mathrm{~nm}$ LED (Cairn Research) on the epifluorescent port of the microscope. A patch pipette filled with ACSF $(1 \mathrm{M} \Omega)$ placed in the stratum radiatum was used as a monopolar stimulating electrode. The recording electrode $(2-3 \mathrm{M} \Omega)$ was filled with the following intracellular medium (in $\mathrm{mM}$ ): $\mathrm{CsMeSO}_{3}$ (125), Hepes (10), EGTA (10), TEA-Cl (8), 4-aminopyridine (5), GTP-Na (0.4), ATP- $\mathrm{Na}_{2}$ (4), $\mathrm{CaCl}_{2}$ (1), and $\mathrm{MgCl}_{2}$ (1) (pH 7.4, $288 \mathrm{mOsm}$ ). Whole-cell patch-clamp recordings were obtained using Axopatch $700 \mathrm{~B}$ (Molecular Devices) and performed in the presence of $10 \mu \mathrm{M}$ bicuculline to block fast inhibitory transmission. Recordings were filtered at $1 \mathrm{kHz}$ and digitized at $10 \mathrm{kHz}$. Paired EPSCs ( $100 \mathrm{~ms}$ interval) were evoked at $0.1 \mathrm{~Hz}$ in neurons held in voltage clamp at $-80 \mathrm{mV}$ to measure AMPAR-mediated EPSCs and then at $+40 \mathrm{mV}$ for NMDAR-mediated EPSCs.

Raw data were analyzed using Clampit (pClamp software version 10.7, Molecular Devices). The amplitude of EPSCs was quantified from the average of 15-30 consecutive responses. The paired-pulse ratio was calculated as the average peak amplitude of the second response divided by that of the first one.

\section{Behavioral experiments}

Behavioral experiments were performed on the injected animals between 2 and 3 months old for open field, self-grooming, marble burying, and rotarod and from 3 to 6-month-old mice for auditory fear conditioning (see the "AAV injections and immunohistochemistry" section for group details). Mice were housed in group cages with 3-6 individuals per cage. Cages were supplemented with minimal enrichment (cotton nestlets). Mice were accustomed to the experimental animal facility and were manipulated every day by the experimenter for at least 1 week before behavioral testing. Behavioral experiments were performed between 8:00 a.m. and 
4:00 p.m. in a light color wall room with light devices. Level of illumination used was as described in Kouser et al. [7]. The experimenter was blind to the genotype and to the material injected during testing.

Open field. Open field consisted of a white square arena $(50 \mathrm{~cm} \times 50 \mathrm{~cm})$. Mice were placed in the center of the arena and left to explore freely for $10 \mathrm{~min}$. Total distance traveled, speed, and time spend in the center zone (defined as a $35 \mathrm{~cm}$ side square) were measured during $10 \mathrm{~min}$ by video tracking (Viewpoint, Lissieu, France).

Self-grooming. Mice were placed in a $25 \mathrm{~cm}$ plexiglass cylinder and video monitored. Time spend self-grooming was visually measured during $10 \mathrm{~min}$.

Marble burying. Mice were placed individually in a clean cage with $10 \mathrm{~cm}$ thick bedding together with 15 marbles evenly distributed on top of the bedding. After $30 \mathrm{~min}$ in the cage, mice were removed and we evaluated the level of marble burying according to the following scores: 0 for a totally buried marble; 1 for a half-buried marble, and 2 for a totally visible marble.

Rotarod. Mice were put on an accelerating rotarod apparatus, $2-45 \mathrm{rpm}$ in $5 \mathrm{~min}$ (Ugo Basile, Gemonio, Italy). Time to fall was measured during four sessions spaced $30 \mathrm{~min}$ apart and the average of the three last trials was measured.

Auditory fear conditioning. We performed an auditory fear-conditioning protocol during 2 consecutive days, using parameters previously optimized in our institute [32]. The experiments were carried out in a fearconditioning apparatus comprising a test box $(20 \mathrm{~cm}$ width $\times 20 \mathrm{~cm}$ length $\times 20 \mathrm{~cm}$ height) placed within a sound proof chamber (Panlab, Harvard Apparatus). Two different contextual configurations were used (A: square configuration, white walls, metal grid floor, washed with $70 \%$ ethanol; B: square configuration, black walls, white floor, washed with $1 \%$ acetic acid). On day 1 , mice were subjected to a habituation session in context $\mathrm{A}$. After $10 \mathrm{~min}$ of habituation to the box, mice received 5 pairing of one tone (CS $+: 2.5 \mathrm{kHz}, 80 \mathrm{~dB}, 10 \mathrm{~s}$ ) with an unconditioning stimulus (US: $0.6 \mathrm{~mA}$ scrambled footshock, $2 \mathrm{~s}$, coinciding with the last $2 \mathrm{~s}$ of CS+ presentation). On day 2, test day, mice were subjected to a $10 \mathrm{~min}$ habituation in context $B$ and then received 12 presentations of the CS+ alone (CS+: $2.5 \mathrm{kHz}, 80 \mathrm{~dB}, 30 \mathrm{~s})$. Freezing behavior during $\mathrm{CS}+$ presentations was analyzed using a load cell coupler (Panlab, Barcelona, Spain) and was defined as the lack of activity above a calibrated threshold for a duration of $2 \mathrm{~s}$ or more as determined with the freezing software (Panlab). To circumvent interferences from locomotion impairment among genotypes on fear responses, we used changes in relative pressure measured during the exploration phase on day 1 to define individual threshold. The average time spent freezing prior to presentation of the sounds (habituation in context B) during the test sessions was used as a measure for contextual fear generalization. We computed a ratio as the fraction of time spent freezing during the $30 \mathrm{~s}$ following $\mathrm{CS}+$ presentation over the fraction of contextual time freezing.

\section{Statistical analysis}

Statistical analysis was carried out using GraphPad Prism 7 software (GraphPad Software Inc.). Statistical tests and post hoc comparisons are indicated in the figure legends and in Supplementary Table S1.

\section{RESULTS}

Transient increase of neuronal activity induces a long-lasting disruption of glutamate receptosome in spines

mGlu5-Homer interaction reports synaptic availability for plasticity induction. The loss of mGlu5-Homer interaction, by preventing mGlu5 and NMDA receptors activation [21, 25], highlights synapses in which induction of plasticity is abolished (Fig. 1A). Recent advances in BRET technology $[26,31]$ allowed us to follow mGlu5-Homer interaction in real time in living hippocampal neurons, to report NMDA synaptic function, and tag synapses that are available for plasticity induction. We fused mGlu5 to the energy donor NLuc and Homer with the fluorescent acceptor Venus. Under stable mGlu5-NLuc expression, the BRET signal increased hyperbolically as a function of the Venus-Homer expression level, indicating a specific interaction between mGlu5 and Homer (Fig. 1B). This BRET signal remained stable over time (Fig. 1C, black, control condition). Neuronal activity modulates the establishment and refinement of neuronal connections, mainly through its effects on dendritic morphology and synaptic plasticity. To determine the effect of neuronal activity on mGlu5-Homer interaction, we treated primary hippocampal cultures with $55 \mathrm{mM} \mathrm{KCl}$ to allow a systematic, wellcontrolled, and long-lasting depolarization of neurons. Neuronal stimulation with $\mathrm{KCl}$ causes an estimated depolarization of $-30 \mathrm{mV}$ (see "Materials and methods"), activation of voltage-gated receptors, and induction of IEGs simulating physiological stimulation [33]. We confirmed IEGs expression induced by this protocol, as illustrated by Homer1a expression (Supplementary Fig. S1). In the neuronal population, depolarization caused a fast decrease of the BRET signal intensity between mGlu5-NLuc and Venus-Homer (Fig. 1C, purple). Alternative protocols to induce plasticity, such as direct stimulation of NMDA receptors, also disrupted mGlu5-Homer interaction (Supplementary Fig. S2). Setting back neurons to their resting membrane potential lead to a partial recovery of the BRET signal (Fig. 1C, orange). Thus, mGlu5-Homer interaction is activity-dependent and only partially reversible upon repolarization. A second subsequent transient increase in neuronal activity fully and reversibly disrupted the recovered mGlu5-Homer complexes (Fig. 1D, red). These data defined two patterns of mGlu5-Homer interaction: one stably disrupted by transient depolarization; a second one highly reversible, which disruption coincides with the cell membrane depolarization. To undergo long-lasting disruption, the first pool of mGlu5-Homer interaction required NMDA receptor activation. In presence of NMDA receptor antagonist, D-AP5, mGlu5-Homer interaction was indeed disrupted by transient depolarization, in a fully reversible manner (Fig. 1C, green). This dichotomy of NMDA receptor involvement, plus the fact that the expression of the glutamate receptosome components are confined to the PSD, supported the hypothesis of regional segregation of the mGlu5-Homer interaction dynamics. In additional support of this hypothesis, BRET imaging demonstrated subcellular specific patterns for mGlu5-Homer interaction dynamics in spines compared to soma (Fig. 1E-H). Images in Fig. $1 \mathrm{E}$ show mGlu5-NLuc expression ( $460 \mathrm{~nm}$ ), Venus-Homer expression (GFP), and the intensities of BRET signal in subcellular compartments (530/ 460). BRET signal was indeed 1.2-fold stronger in spines than in soma (Fig. 1F). In the soma, we measured a homogenous mGlu5-Homer interaction, which was disrupted by transient depolarization and fully reversed within $10 \mathrm{~min}$ (Fig. 1G). In contrast, we noticed an important heterogeneity between spines and divided them into two equivalent populations depending on their BRET signal intensities (Fig. 1H). Importantly, the higher the BRET signal was, the stronger the efficiency of neuronal activity to disrupt the interaction. In these spines, the transient depolarization irreversibly disrupted mGlu5-Homer interaction (Fig. 1H, red). Low basal BRET signal in spines may reflect recent synaptic activation. Consistently, no significant change in BRET was recorded in these spines following transient depolarization (Fig. 1 H, blue). Altogether, these experiments show that the availability of synapses for plasticity induction, as reported by mGlu5-Homer interaction, is heterogenous among spines and is blocked at least for $1 \mathrm{~h}$ following transient depolarization.

\section{Glutamate receptosome disruption decreases NMDA/AMPA excitatory postsynaptic currents ratio and impairs plasticity induction}

Spines containing undisrupted glutamate receptosome (high basal BRET, Fig. $1 \mathrm{H}$ ) undergo activity-driven NMDA receptor activation, followed by long-lasting complex disruption. Highly permeable to calcium ions, NMDA receptors play a key role in activity-induced long-term changes of synaptic strength. mGlu5 direct interaction with NMDA receptors enables mutual functional inhibition $[21,25]$. We also know that Homer isoforms control mGlu receptor localization [34]. Therefore, scaffolding complex 
disruption could enhance mGlu5 receptor mobility enabling its direct interaction with and inhibition of NMDA receptor. This was indeed supported by our previous report using single-molecule tracking, where mGlu5 was significantly more mobile at synapses in hippocampal neurons when mGlu5-Homer interaction was disrupted, causing an increased synaptic surface coclustering of mGlu5 and NMDA receptors [35]. Consistently, we found that a transient increase in neuronal activity, inducing disruption of glutamate receptosome (Fig. 1), enhanced endogenous mGlu5 mobility (Supplementary Fig. S3). To further understand the role of glutamate receptosome dynamics on glutamatergic synapse physiology, we disrupted mGlu5-Homer interaction and studied the functional consequences on NMDA/AMPA excitatory postsynaptic current ratio and on the activation of ERK and mTOR pathways as readouts of synaptic plasticity. We used a cellpermeant TAT peptide containing the proline-rich motif of the mGlu5 C-terminal tail (TAT C-tail) to compete with mGlu5 to bind the EVH1 domain of Homer [28]. TAT C-tail disrupted mGlu5-Homer interaction in less than $10 \mathrm{~min}$, while a control TAT peptide containing a mutated Homer-binding motif had no significant effect (Supplementary Fig. S4).

The NMDA/AMPA ratio is an important parameter that strongly influences the integrative properties of excitatory synapses. Acute treatment of brain slices with the TAT C-tail (but not TAT control) induced a strong decrease of the NMDA/AMPA postsynaptic current ratio in the hippocampus CA1 (Fig. 2A). Therefore, depolarization-induced glutamate receptosome disruption would increase mGlu5 mobility, leading to feedback inhibition of NMDA activity.

We then studied the activation of ERK and mTORC1 pathways, in real time, using specific intramolecular BRET-based biosensors in hippocampal neuronal cultures (YEN [26] or AIMTOR [27], respectively, Fig. 2B). Transient depolarization enhanced ERK (Fig. 2C, TAT ctrl) and mTOR (Fig. 2D, TAT ctrl) signaling pathways. Interestingly, disrupting glutamate receptosome prevented both pathways activation by a subsequent depolarization (Figs. 2C, D, TAT C-tail). Altogether, these results show that impaired glutamate receptosome dynamics alter mGlu5 mobility, NMDA/AMPA ratio, and synaptic plasticity. These data explain how disruption of glutamate receptosome prior to changes of neuronal activity prevents NMDA-dependent plasticity induction [21]. Hence, a first neuronal activation inducing NMDA-dependent plasticity, by disrupting the complex in a long-lasting manner and inducing feedback inhibition of NMDA receptors, would freeze synaptic sensitivity to subsequent neuronal activation, allowing the expression of the initiated plasticity.

\section{NMDA/AMPA excitatory postsynaptic currents ratio and neuronal activity-induced signaling are altered in Shank3 $\Delta C$ mice}

In Shank $3 \Delta \mathrm{C}$ mice, a truncated mutant form of Shank3 prevents the integrity of the glutamate receptosome. As glutamate receptosome remodeling after depolarization controls NMDA/ AMPA postsynaptic excitatory currents ratio and ERK/mTOR activation pathways, we studied these functional aspects in Shank $3 \Delta C$ mice. We observed a significant decrease in the NMDA/AMPA ratio in the CA1 area of the hippocampus of Shank3 $\triangle C$ mice compared to WT mice (Fig. $3 A$ ), confirming previous studies in hippocampus [7] and cortex [8]. We noticed an important variability of the NMDA/AMPA ratio in WT mice, probably due to the developmental window of the experiment (P15-P20) during which synapses are maturing, while this ratio was much less variable in Shank3 $\Delta \mathrm{C}$ mice. We found no difference in the paired-pulse ratio between Shank $3 \Delta C$ mice and WT mice, arguing for a postsynaptic deficit rather than presynaptic (Fig. 3A). Furthermore, Shank3 $\Delta \mathrm{C}$ mice displayed strong impairment in ERK and mTOR activation by transient depolarization (Fig. 3B, C, respectively). We have previously shown that glutamate receptosome disruption, by enabling mGlu5 and NMDA receptor interaction and mutual blockade, prevents induction of plasticity [21]. Interestingly, coimmunoprecipitation experiments (Fig. 3D) revealed an increased association between endogenous mGlu5 and NMDA receptors in Shank3 $\Delta C$ mice hippocampi compared to WT mice. Importantly, total amount of GluN1 protein was unchanged between WT and Shank $3 \Delta \mathrm{C}$ mice (Supplementary Fig. S5), as previously published [7]. These results suggest that decreased NMDA/AMPA current ratio and ERK/mTOR signaling impairments in Shank $3 \Delta \mathrm{C}$ mice are likely due to molecular dynamics deficiencies of the glutamate receptosome. Hence, we assessed mGlu5-Homer dynamics by BRET in neurons from Shank3 $\Delta C$ mice compared to WT (Fig. $3 E$ ). In neuronal population, we found no difference in the mGlu5-Homer disruption induced by depolarization between the two genotypes (purple curves) but a significant increase in BRET signal recovery after repolarization (orange curves) in Shank3 $3 \mathrm{C}$ mice compared to WT mice. Indeed, only $36.1 \% \pm 6.9 \%$ of mGlu5-Homer disruption was long lasting in Shank $3 \Delta \mathrm{C}$ mice versus $53.4 \% \pm 3.9 \%$ in WT mice (Fig. $3 \mathrm{E}$, right panel). These results show that in Shank3 $\Delta \mathrm{C}$ mice, mGlu5-Homer interaction is still sensitive to neuronal activity but remains highly reversible, fluctuating with membrane depolarization. A second subsequent transient increase in neuronal activity indeed disrupted mGlu5-Homer interaction again in Shank $3 \Delta \mathrm{C}$ neurons (Fig. 3F). These longer lasting experiments further underline the better recovery of the BRET signal in Shank $3 \Delta \mathrm{C}$ neurons compared to WT neurons (see Fig. 1D). In addition, BRET imaging showed that more than $90 \%$ of the Shank $3 \Delta \mathrm{C}$ spines express high basal BRET values between mGlu5 and Homer (Fig. 3G). In these spines, mGlu5-Homer interaction can be broken by depolarization and restored by repolarization (Fig. $3 \mathrm{H}$ ), contrary to WT spines (Fig. $1 \mathrm{H}$ ). Altogether, these experiments show that a dysregulation of mGlu5 receptosome dynamics in Shank3 $\Delta C$ mice is associated with alterations of NMDA/AMPA current ratio and ERK/mTOR signaling.

\section{Repairing scaffolding complex dynamics rescues activity- induced signaling in Shank3 $\Delta \mathrm{C}$ hippocampal neurons}

In Shank $3 \Delta \mathrm{C}$ mice, the mGlu5-Homer interaction is still regulated by neuronal activity but only in a short-lived manner, having no more functional consequences on neurotransmission and plasticity. Therefore, glutamate receptosome integrity would be required for synaptic plasticity induction and expression. To test this hypothesis, we generated a molecular bridge between mGlu5 and NMDA receptors, bypassing Shank3, by fusing Homer to the C-tail PDZ ligand of GluN2B NMDA subunit after a glycine linker [36], (Fig. 4A). Homer-GluN2B chimaera was correctly expressed and targeted at the spines of Shank3 $\Delta C$ neurons (Fig. 4B) and colocalized with PSD95 (Supplementary Fig. S6). BRET experiments in Shank3 $\Delta C$ neuronal population showed that Homer-GluN2B chimaera interacts with mGlu5 in a neuronal activity-dependent manner (Fig. 4C), recapitulating functional features of Homer-mGlu5 interaction in WT mice. Indeed, restoring the glutamate receptosome integrity by expressing the Homer-GluN2B chimaera rescued the long-lasting disruption induced by the neuronal activity in Shank $3 \Delta \mathrm{C}$ neurons at $49.3 \% \pm$ 9.3\% (compared to $53.4 \% \pm 3.9 \%$ in WT and $36.1 \% \pm 6.9 \%$ in Shank3 $\Delta$ C, Fig. 3E).

BRET experiments in Shank $3 \triangle \mathrm{C}$ neurons expressing Homer-GluN2B chimaera revealed a strong increase of ERK and mTOR activation induced by transient depolarization (Fig. 4D, E, respectively). We noticed a significant decrease of the basal AIMTOR BRET signal intensity in Shank3 $\Delta C$ neurons expressing Homer-GluN2B chimaera compared to Shank $3 \Delta C$ neurons. Thus, the inability of Shank $3 \Delta C$ neurons, before rescue, to activate the mTOR pathway in response to the neuronal activity could be due to mTOR hyperactivation in basal conditions (Fig. 4E). Altogether, these experiments show that restoring glutamate receptosome dynamics rescued neuronal signaling and synaptic plasticity in Shank $3 \Delta C$ mice. Of note, we found that in Shank3-depleted 
A
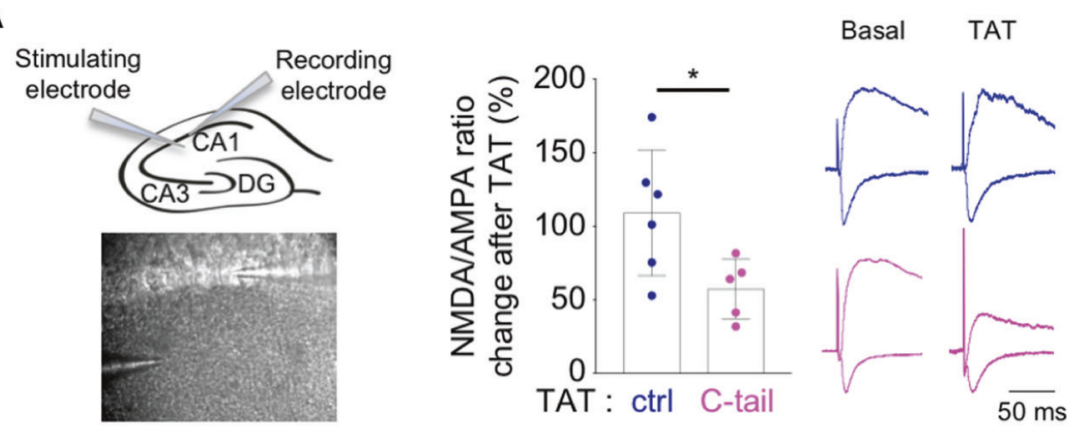

B

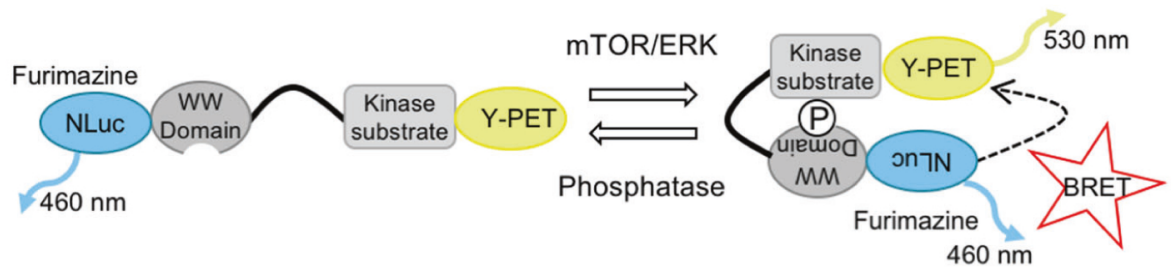

C ERK activation
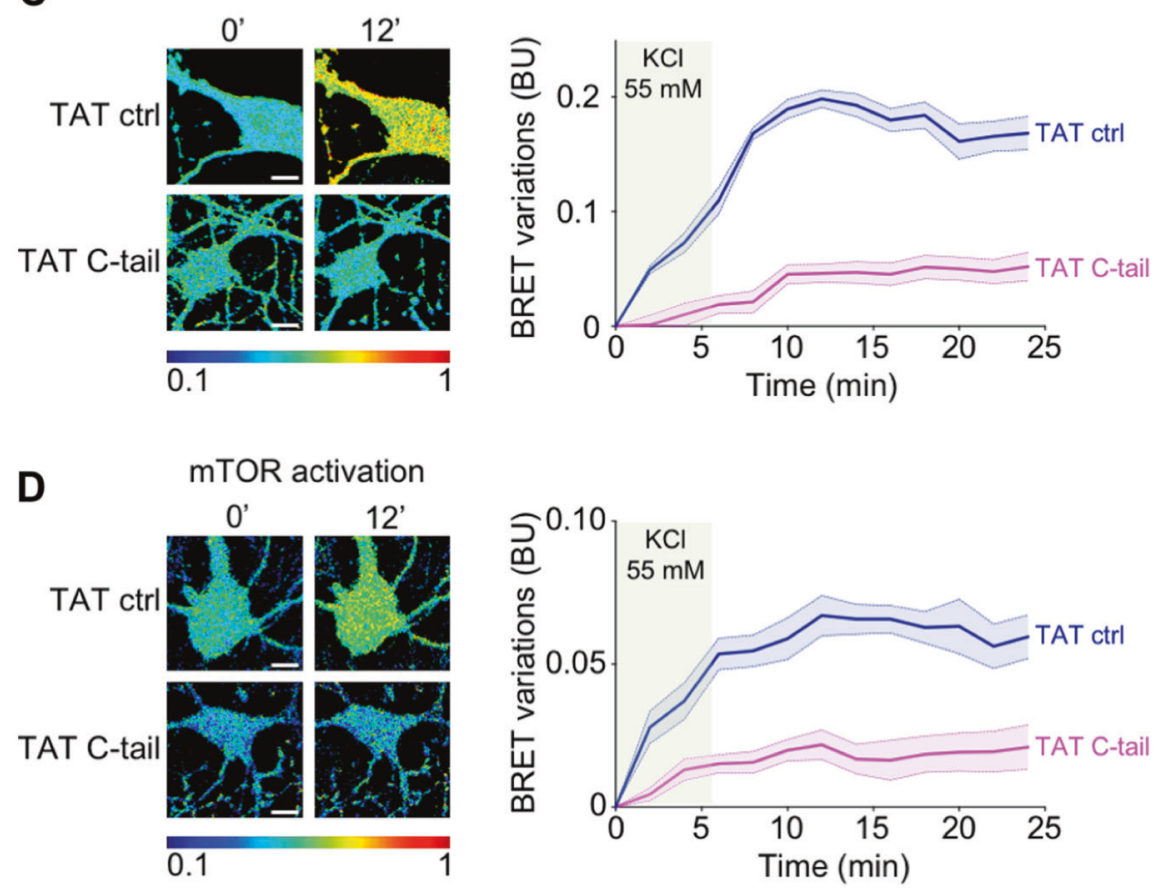

Fig. 2 Acute mGlu5-Homer disruption impairs NMDA/AMPA postsynaptic currents ratio and ERK and mTOR signaling pathways. A CA1 pyramidal neurons were clamped at -80 and $+40 \mathrm{mV}$ to record AMPA and NMDA currents, respectively, before and $10 \mathrm{~min}$ after perfusion of TATs (ctrl or C-tail). For each neuron, the NMDA/AMPA ratio is expressed as the percentage of NMDA/AMPA ratio before TAT perfusion. $N=5-6$ neurons from three to four mice. Right panel: representatives average traces at -80 and $+40 \mathrm{mV}$ of the same recording before and $10 \mathrm{~min}$ after TAT perfusion. B Schematic representation of the conformational changes of mTOR or ERK BRET biosensors upon their activation. BRET variations in neurons expressing YEN (C) or AIMTOR (D) biosensors before $\left(0^{\prime}\right)$ and 12 min after depolarization with $\mathrm{KCl} 55 \mathrm{mM}\left(12^{\prime}\right)$. Cultures were supplemented with TATs (ctrl or C-tail) 30' before recordings (8-30 neurons, three independent experiments). Scale bar 10 $\mu$ m. A-D Data are mean \pm SEM; * indicate $p$ value $<0.05$; Mann-Whitney test. C, D Point-by-point statistical tests and post hoc comparisons are indicated in Supplementary Table S1.

neurons using a specific shRNA, transfected Homer-GluN2B chimaera is correctly recruited to synapses while transfected WT Homer is not (Supplementary Fig. S7). Hence, our strategy of bypassing Shank3 mutations/absence by reconstruction of a molecular bridge between metabotropic and ionotropic glutamate receptors with Homer-GluN2B chimaera could be extended to other Shankopathies.
Repairing scaffolding complex dynamics rescues NMDA/AMPA excitatory postsynaptic currents ratio and improves cognitive impairments in Shank3 $\Delta \mathrm{C}$ mice

We next investigated whether repairing scaffolding complex dynamics with Homer-GluN2B chimaera could rescue cognitive impairments in Shank $3 \Delta C$ mice, in vivo. We bilaterally injected the lateral ventricles of $P 7$ Shank $3 \triangle C$ mice with $A A V$ encoding 
A
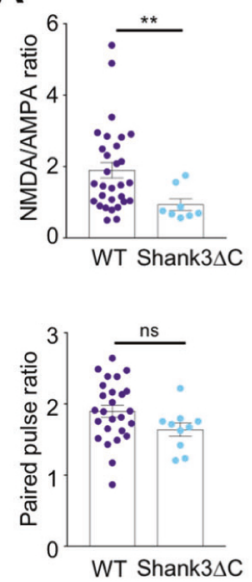

D
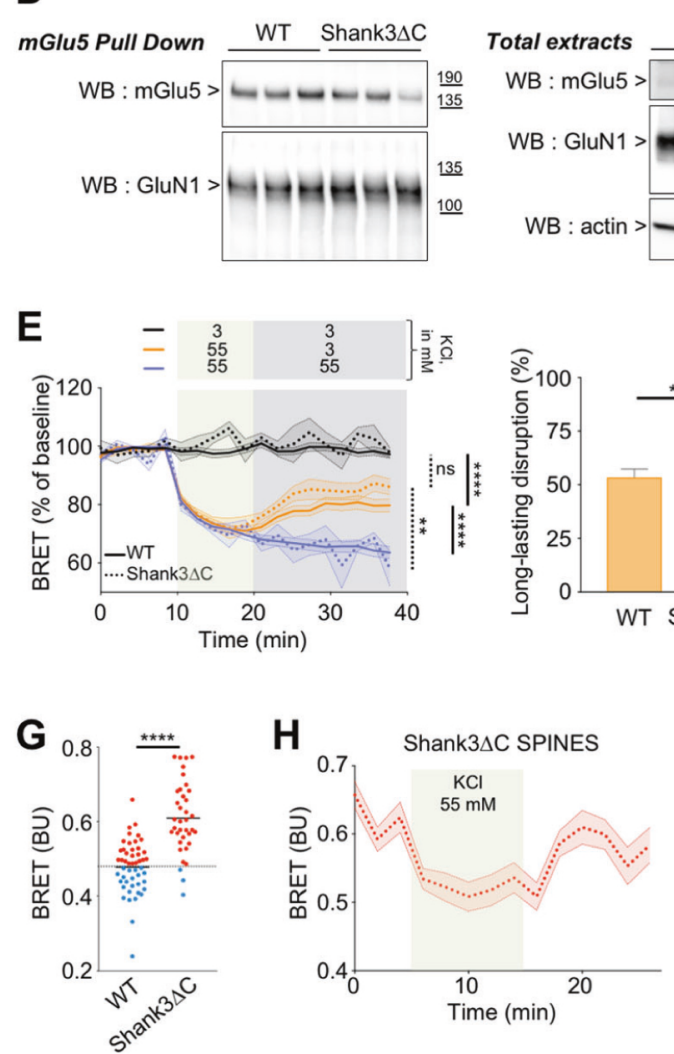

B ERK activation
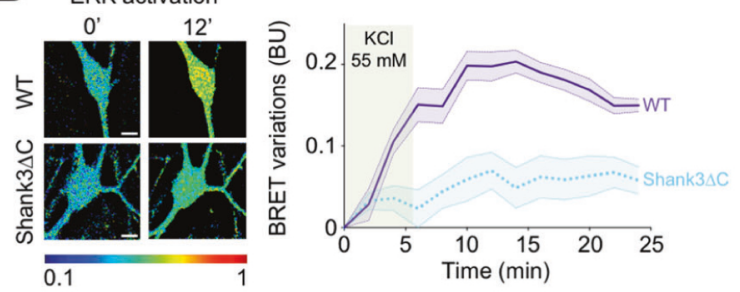

C
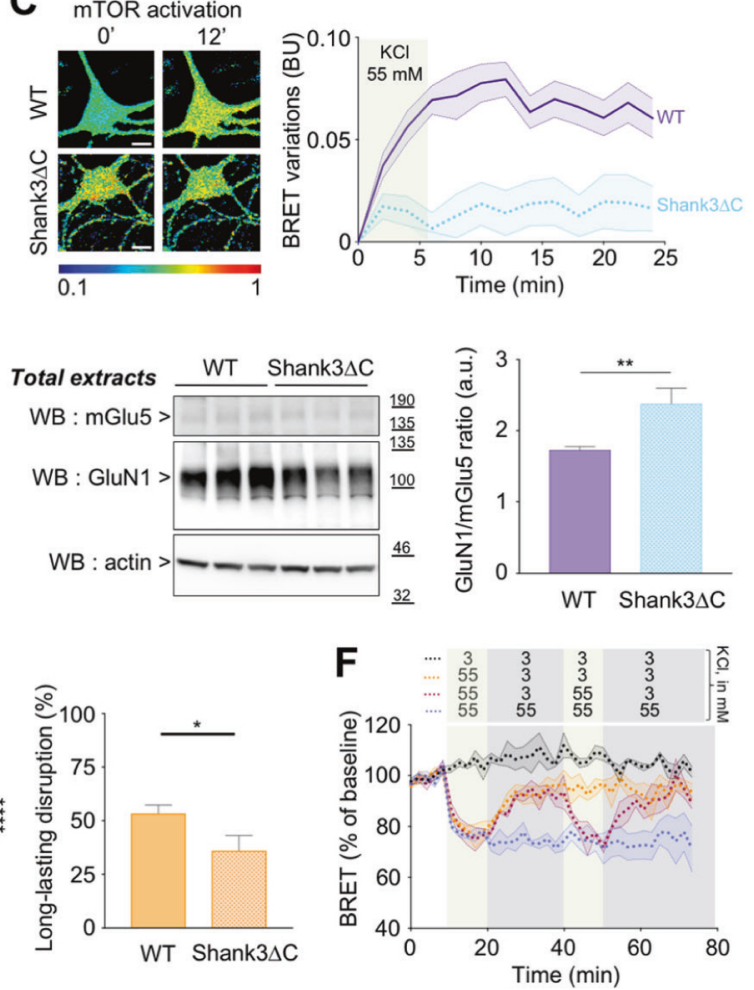

Fig. 3 NMDA/AMPA currents ratio, ERK and mTOR signaling pathways, and mGlu5-Homer interaction are altered in Shank3 $\triangle \mathrm{C}$ mice. A CA1 pyramidal neurons from WT or Shank $3 \Delta \mathrm{C}$ mice were clamped at -80 and $+40 \mathrm{mV}$ to record AMPA and NMDA currents, respectively (100 ms interstimulus). $N=8-30$ neurons from 3 to 15 mice. Right panels: representatives average traces at -80 and $+40 \mathrm{mV}$. Scale bars $50 \mathrm{pA}$ over 50 ms. BRET variations in WT or Shank3 $\Delta$ C neurons expressing YEN (B) or AIMTOR $(\mathbf{C})$ biosensors before $\left(0^{\prime}\right)$ and $12^{\prime}$ after depolarization $(8-30$ neurons, three independent experiments). Scale bar $10 \mu \mathrm{m}$. D Left panel shows immunoblotting for mGlu5 and GluN1 after mGlu5 pull-down. Middle panel shows total lysates immunoblotted for mGlu5, GluN1, and actin. Graph presents the GluN1/mGlu5 ratio. BRET variations (WT = line; Shank3 $\Delta C=$ dotted line) during membrane potential changes (at least three independent experiments), recorded with a fluorometer ( $E$, F) or under microscope $(\mathbf{G}, \mathbf{H})$. E (left) One-way ANOVA multiple comparison with Tukey correction on the averaged last two points of each curve. Dotted/full lines to compare Shank3 $\Delta \mathrm{C} / \mathrm{WT}$ conditions, respectively. $\mathbf{E}$ (right) Long-lasting disruption calculated on the averaged last two orange time points, expressed as a percentage of respective blue time points. G Distribution of the basal BRET intensities in spines, WT, and Shank3 $\Delta \mathrm{C}$ mice. The dashed line indicates the median of basal BRET intensities in spines from WT mice (0.48). H Over 37 spines (15 neurons, three independent experiments), the 34 having high basal BRET signal $(>0.48)$ are represented. Only three spines displayed a low basal BRET $(<0.48)$ and were not represented. A-H Data are mean \pm SEM; ns, ${ }^{*}, * * * * * *$ indicate $p$ value $>0.05,<0.05,<0.01,<0.0001$, respectively; Mann-Whitney test (excepted for left E). Point-by-point statistical tests and post hoc comparisons for $\mathbf{B}, \mathbf{C}$, E, $\mathbf{F}$, and $\mathbf{H}$ are indicated in Supplementary Table S1.

Homer-GluN2B chimaera tagged with a green fluorescent protein or only eGFP as a control. We also injected WT littermates with AAV-expressing eGFP (Fig. 5A). AAV injection triggered a high transgene expression mainly in the hippocampus. Other structures such as cortex, striatum, thalamus, and amygdala express the transgene with few heterogeneities among animals (Supplementary Fig. S8). Importantly, Homer-GluN2B chimaera rescued Shank3 $\Delta C$ synaptic transmission deficit, as shown by 
A

Restoring glutamate receptosome in Shank3 $\Delta \mathrm{C}$ mice
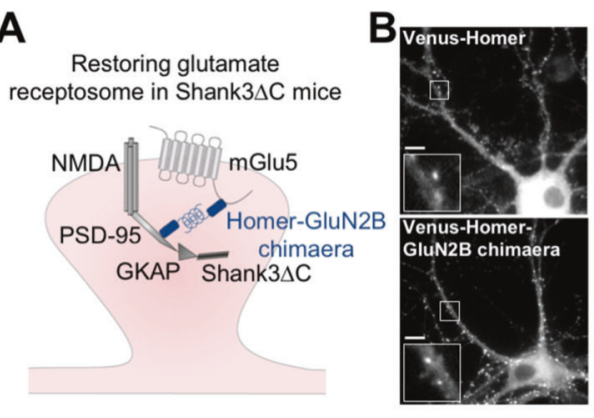
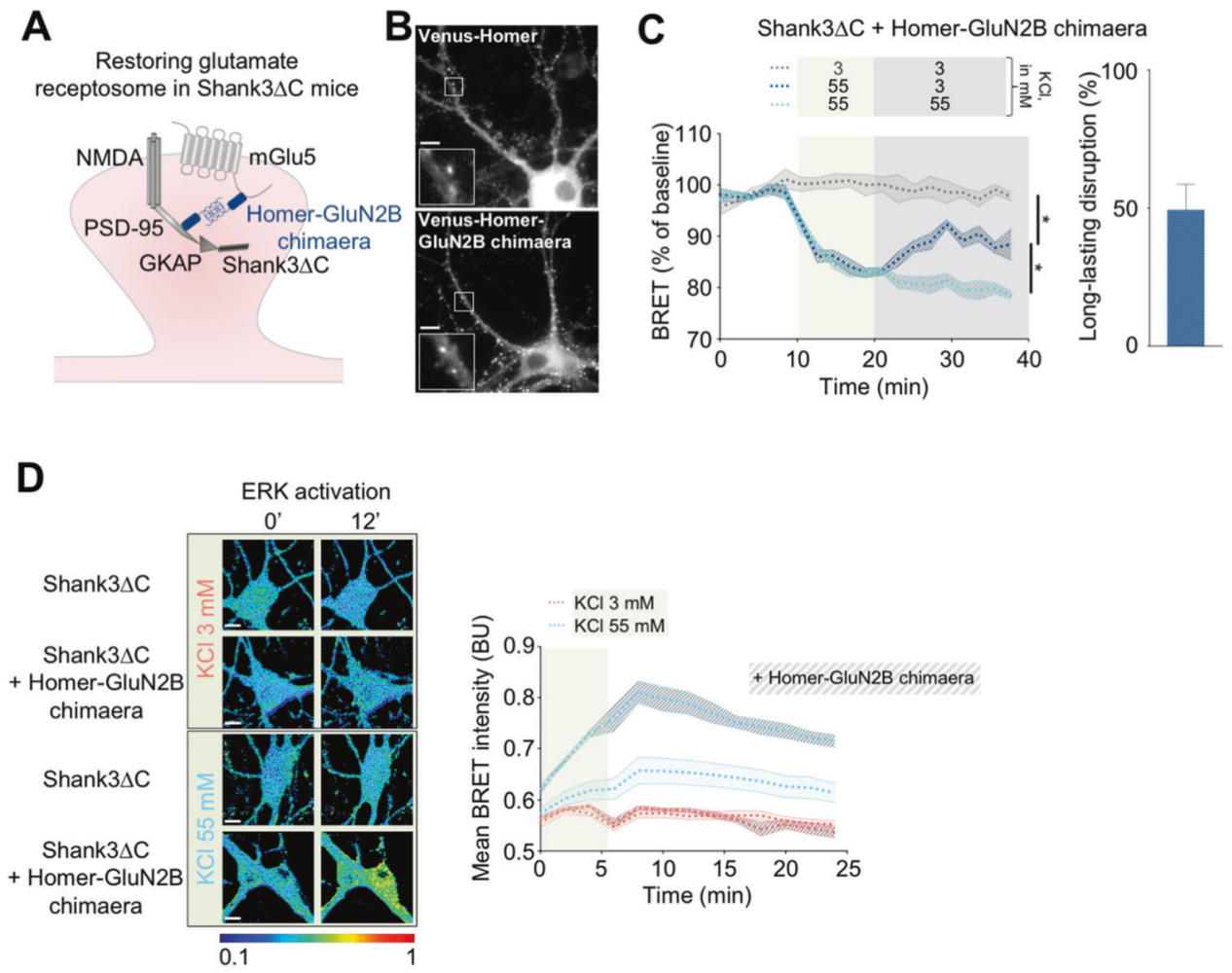

$\mathbf{E}$
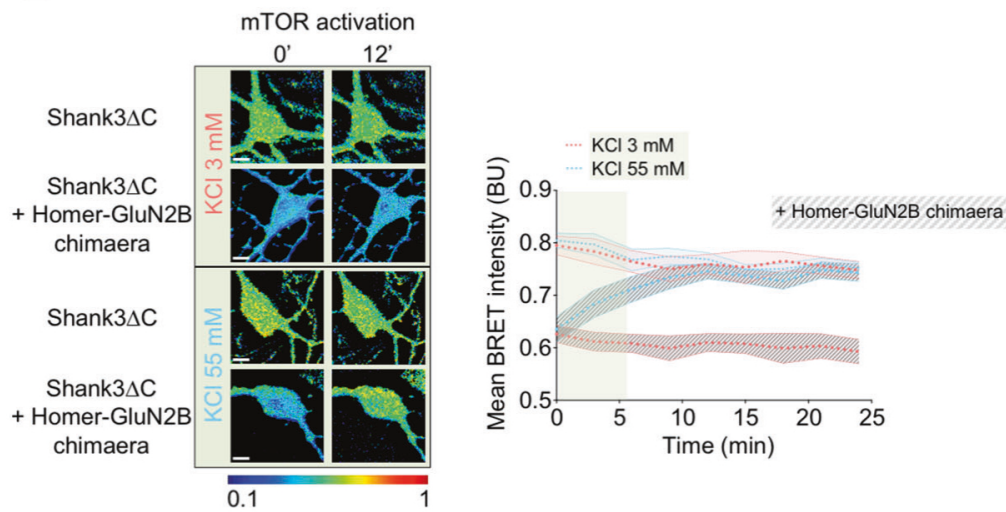

Fig. 4 Repairing glutamate receptosome dynamics rescues ERK and mTOR signaling pathways in Shank3 $3 \mathbf{C}$ mice. A Schematic illustration of the glutamate receptosome reparation in Shank3 $\Delta C$ neurons by expressing Homer-GluN2B chimaera to rescue synaptic availability for plasticity induction. B Comparison of Homer and the Homer-GluN2B chimaera expression in Shank3 $\Delta C$ hippocampal neurons. Scale bar $10 \mu \mathrm{m}$. C BRET variations between mGlu5-NLuc and Venus-Homer-GluN2B chimaera in Shank3 $\Delta C$ neurons. Data are mean \pm SEM of BRET recorded from at least four independent cultures. One-way ANOVA multiple comparison statistical analysis with Tukey correction to compare the last two time points of each curve. * indicates $p$ value $<0.05$. Right: Long-lasting disruption calculated on the averaged last two indigo time points, expressed as a percentage of respective cyan time points. BRET variations in Shank3 $\Delta C$ neurons expressing YEN (D) or AIMTOR (E) biosensors before $\left(0^{\prime}\right)$ and $12 \mathrm{~min}\left(12^{\prime}\right)$ after depolarization. Scale bar $10 \mu \mathrm{m}$. Data are mean \pm SEM of BRET intensities recorded in 8-30 neurons from three independent experiments. Point-by-point statistical tests and post hoc comparisons for $\mathbf{C}-\mathbf{E}$ are indicated in Supplementary Table 1.

measurement of NMDA/AMPA excitatory postsynaptic current ratio (Fig. 5B). Of note, NMDA/AMPA ratio was not affected by the injection of the control AAV (Figs. 3A and $5 B$ ), neither in WT nor in Shank3 $\triangle C$ mice ( $p$ value comparing mice injected or not with AAV ctrl: 0.4 in WT and 0.5 in Shank3 $\Delta$ C; Mann-Whitney test), showing that surgery and viral expression had no effect per se on synaptic transmission. We further assessed the consequences on behaviors by performing a broad series of test in which Shank $3 \Delta \mathrm{C}$ mice are known to present deficits [7, 8, 10], from locomotion to stereotyped behaviors and anxiety. We also tested Shank $3 \Delta C$ mice in a fear-conditioning paradigm. Compared to WT littermates, Shank $3 \Delta C$ mice traveled less distance and with a lower speed in the open field test (Fig. 5C). This aberrant locomotion was confirmed by their poor performance in the accelerating rotarod (Fig. 5D) and not rescued by Homer-GluN2B chimaera expression. Shank $3 \Delta \mathrm{C}$ mice also present increased anxiety-like behaviors with a decreased time spent in the center during the open field test (Fig. 5C), an increased time spent self-grooming (Fig. 5E) and a higher score during fear conditioning (Fig. 5F). The self-grooming test is also indicative of repetitive stereotyped behaviors in Shank $3 \Delta$ C mice. Importantly, Homer-GluN2B chimaera expression in Shank $3 \Delta C$ mice rescued these anxiety-like and stereotyped behaviors in these three behavioral tests (Fig. 5C, E, F). In the fearconditioning test, Shank $3 \Delta \mathrm{C}$ mice have a higher score compared 
A

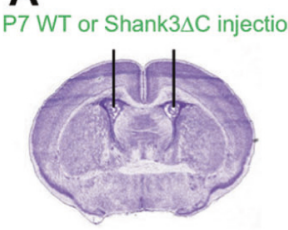

Adult Shank3 $\Delta C$

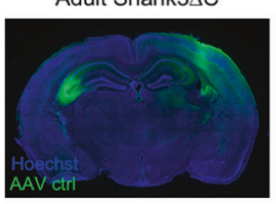

C

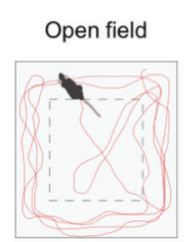

$10^{\prime}$

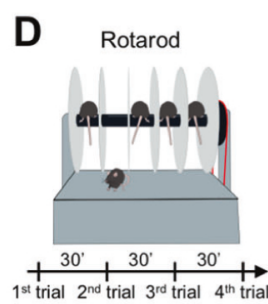

F

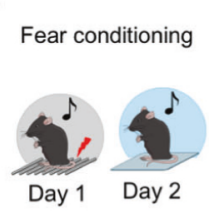

Adult WT

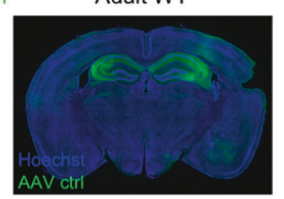

Adult Shank3 $\Delta C$

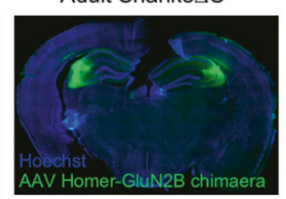

B

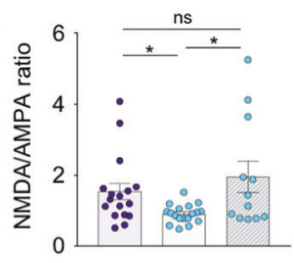

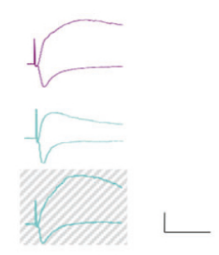

- $W T+$ AAV ctrl

- Shank3 $\triangle \mathrm{C}+\mathrm{AAV}$ ctrl or AAV Homer-GluN2B chimaera
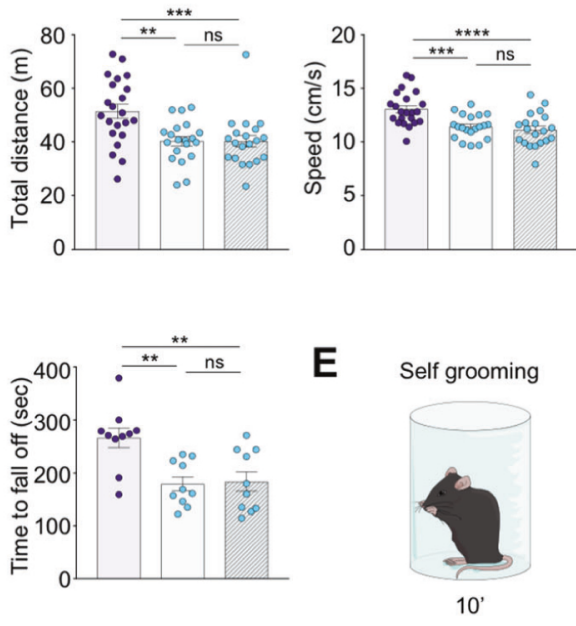

E

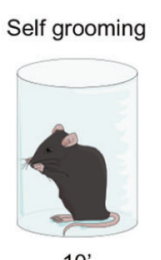

10

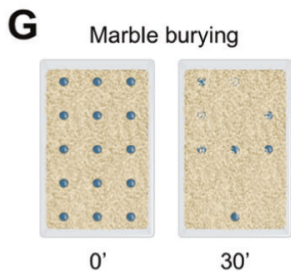

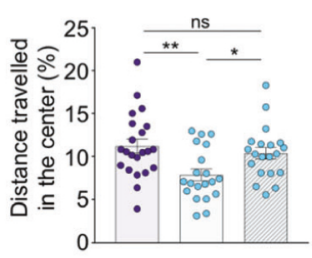

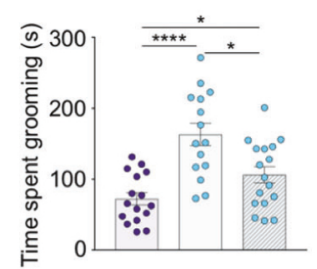

E

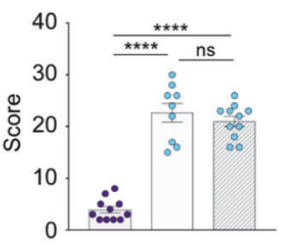

Fig. 5 in vivo glutamate receptosome dynamics reestablishment rescues NMDA/AMPA currents and autistic-like behaviors in Shank3 $\triangle C$ mice. A Schema of injection and representative images of a coronal brain slice for each group. B-G Data are mean \pm SEM. ns not significant; $q$ value: ${ }^{*}<0.05 ;^{* *}<0.01 ;{ }^{* * *}<0.001 ; * * * *<0.0001$; Kruskal-Wallis test with FDR post-test. B Green CA1 pyramidal neurons from injected WT or Shank3 $3 \mathrm{C}$ mice were clamped at -80 and $+40 \mathrm{mV}$ to record AMPA and NMDA currents, respectively. $N=12-18$ neurons from 5-7 mice per condition. Right panel: representatives average traces at -80 and $+40 \mathrm{mV}$. Scale bar from the top to the bottom: 70,60 , and $40 \mathrm{pA}$ over $50 \mathrm{~ms}$. C-G $N=8-17$ mice per group. C Open field. Left panel: Top view of the arena with an illustration of a mouse trajectory during 10 min exploration. Dashed line square represents the center zone. Total distance and speed are measured during 10 min. Right panel: Percentage of distance traveled in the center zone with respect to the total distance traveled during 10 min. D Left panel: Accelerating rotarod apparatus and protocol. Right panel: Time in seconds to fall from the apparatus (average of the three last trials). E Left panel: Illustration of the selfgrooming plexiglass cylinder in which grooming was quantified. Right panel: Time spent grooming by each group. $\mathbf{F}$ Fear conditioning. Left panel: Illustration showing the protocol. Right panel: Ratio of time spent freezing after CS+ over contextual time spent freezing. G Marble burying. Left panel: Illustration showing the initial marble disposition and an example of buried marbles after 30 min. Right panel: marble burying score quantification per group.

to WT mice. This score is the ratio of time spent freezing following $\mathrm{CS}+$ presentation over contextual habituation and is rescued in the Shank3 $\Delta$ C Homer-GluN2B chimaera group. In accordance with other Shank3 mutant mice studies [37-41], Shank3 $\Delta \mathrm{C}$ mice show no deficit in aversive learning, but display altered level of fear expression during the different phases of the test (Supplementary Fig. S9). This could be the result of a combination of an altered locomotion and an increased level of anxiety. While the marble burying is usually used to test anxiety, it was recently described to test avoidance behavior to novelty in Shank3 $\Delta C$ mice [7] who show no interest in burying marbles compared to WT littermates (Fig. 5G). This behavior is unchanged in Shank3 $\Delta \mathrm{C}$ mice injected with Homer-GluN2B chimaera. Altogether, these experiments show that Shank $3 \Delta C$ mice present deficits in locomotion, an avoidance behavior to novelty, anxiety-like behaviors, and repetitive stereotyped behaviors. Injecting Homer-GluN2B chimaera in the lateral ventricles of Shank $3 \Delta C$ mice decreased anxiety-like and repetitive stereotyped behaviors, suggesting that a deficit in glutamate receptosome dynamics could be at the origin of the main core symptoms in ASD. 


\section{DISCUSSION}

Shankopathies are one of the most common genetic causes of ASD [4, 5]. A deficit in NMDA neurotransmission represents a patho-physiological hallmark underlying cognitive impairments in Shankopathies and other ASD such as Fragile $X$ syndrome. However, very little is known about the molecular synaptic mechanisms leading to this NMDA receptor impairment. In the past decade, we and others have highlighted the key role of mGlu5-Homer interaction in the control of synaptic plasticity and cognition. Indeed, following a first induction of NMDA-dependent plasticity, a decrease of mGlu5-Homer interaction is associated to physical interaction between mGlu5 and NMDA receptors and feedback inhibition of NMDA currents, preventing subsequent induction of plasticity [21]. In other words, scaffold remodeling controls availability of synapses for induction of plasticity. Here, we used mGlu5-Homer interaction to report this synaptic availability by BRET. We depicted an activity-driven dynamic remodeling of mGlu5-Homer interactions, in WT mice. Transient neuronal depolarization, activating NMDA receptors to induce plasticity, causes a rapid break in the mGlu5-Homer interaction (within tens of seconds) while fundamental signals for synaptic plasticity expression, such as mTOR and ERK pathways are increased sustainably. In spines, this disruption is not reversed, possibly for hours. During this phase of plasticity expression, the scaffolding complex is broken and the NMDA receptors are blocked, making the synapse unavailable for subsequent plasticity induction. Indeed, a sustained increase in neuronal activity while mGlu5-Homer interaction is broken is no longer able to induce molecular and cellular plasticity. Interestingly, we observed the same kind of defects in the Shank3 $\Delta C$ mouse model of $A S D$, in which a truncated form of Shank3 prevents scaffolding complex integrity. Shank $3 \Delta \mathrm{C}$ neurons indeed display a decreased basal NMDA/AMPA postsynaptic current ratio associated with a strong impairment in activity-driven increase of ERK and mTOR signaling pathways. We also confirmed previous reports, showing that these Shank3 $\Delta C$ mice display strong anxiety-related and repetitive stereotyped behaviors $[7,8,10]$. We then repaired the molecular synaptic complex in Shank3 $\Delta \mathrm{C}$ mice, while preserving its sensitivity to neuronal activity. Interestingly, this gene therapy restored the cellular plasticity and relieved stereotyped and anxiety-related behaviors in the Shank $3 \Delta C$ mice. Altogether, our data support a new theory according to which the glutamate receptosome complex, by its capacity to be reshaped by the neuronal activity, modifies NMDA receptors synaptic function and hence plays a decisive role in metaplasticity by sliding the induction threshold for plasticity. We further propose that molecular synaptic deficiencies would trigger synaptic plasticity impairments and atypical cognitive behaviors, reminiscent of ASD. This work opens opportunities for gene therapy strategies.

The first part of our study unveils mGlu5-Homer interaction dynamics in living cells upon neuronal activation. Neuronal depolarization induces a fast disruption of this interaction, uniformly in all neuronal subcellular compartments. However, the ability of the complex to re-form differs between subcellular compartments. In the soma, mGlu5 and Homer interact again as soon as the membrane potential resets back to resting potential. Successive transient depolarizations break and re-form the interaction, fleetingly. To date we know neither the molecular mechanisms sensing depolarization nor the functional consequences of this ephemeral remodeling. Future studies will be required to better understand this phenomenon. In contrast, in half of the spines, the transient depolarization brakes mGlu5-Homer interaction within tens of seconds, in a longlasting manner. We also show that NMDA receptors activation by neuronal depolarization is necessary to induce long-lasting disruption. The fast disruption between Homer and mGlu5 could be attributed, at least in part, to $\mathrm{Ca}^{2+}$ entry through NMDA receptors. $\mathrm{Ca}^{2+}$ would activate CamKIla which can phosphorylate the long isoform of Homer, reducing its interaction with mGlu5 [42]. The IEG Homer1a, whose expression is induced by the increased neuronal activity, is then favored in the competition with the long form of Homer to interact in its place with the mGlu5 receptor [21]. This could explain the long-lasting disruption of mGlu5-Homer interaction. This stably disrupted conformation of the receptosome could be a homeostatic mechanism to make synapses undergoing plasticity unavailable for further stimulation to induce new plasticity. We indeed show a strong heterogeneity in mGlu5-Homer dynamics in the spines that are attributable to differences in the basal interaction between these two proteins. The mGlu5-Homer disruption is almost absent in spines where the interaction is already weak. We can easily speculate that the BRET signal is low in these spines either because they are immature or because they recently underwent plasticity. Hence, the synaptic availability defined as the readiness of the synapse to a stimulus would be temporally blocked. This would favor the long-term expression of the initiated plasticity. These molecular dynamics at synapses explain reported roles of mGlu5-Homer in metaplasticity [43-45]. Importantly, all these studies report Homer1a as the dominant-negative IEG breaking mGlu5-Homer interaction to allow synaptic plasticity. However, our experiments here demonstrate that mGlu5-Homer interaction is disrupted in few seconds while Homer1a synthesis takes about 20 min [46]. This suggests that mGlu5-Homer interaction disruption could be the synaptic tag to attract Homer1a in potentiated synapses. This could be a homeostatic mechanism to lock a spine undergoing plasticity into a functional network, by decreasing temporarily its availability for plasticity induction. This hypothesis calls for further study, to know whether and by which mechanisms a spine can be "unlocked" from a functional network.

The molecular cascade triggered by NMDA-dependent neuronal activation in physiological conditions includes scaffold disruption, increase of mGlu5 mobility, enhancement of mGlu5-NMDA clustering, inhibition of NMDA receptor activity, and decrease of the NMDA/AMPA ratio. These molecular events enable induction of ERK and mTOR activation. ERK activation is common to many forms of synaptic plasticity, in particular NMDA-dependent LTP in many brain areas $[47,48]$. mTOR also sustains plasticity, memory storage, and cognition (see reviews $[49,50])$. While the important role of ERK and mTOR signaling pathways in neuronal plasticity has long been known, very little is known concerning the activation kinetics of these signaling pathways due to the lack of real-time experimental approaches. Here, we used mTOR and ERK biosensors [26, 27] and showed that both kinases are immediately activated by transient increase of neuronal activity to reach a maximum 5-7 min later. Surprisingly, phosphorylation of both ERK and mTOR substrates lasted for more than $20 \mathrm{~min}$. These biosensors integrate kinases and phosphatases activities. This long-lasting substrate phosphorylation could report either kinases long-lasting activation or phosphatases inhibition during the late phase of neuronal plasticity. The later hypothesis is supported by previous findings, showing that activation of mTOR is required only during the early phase of LTP [51]. Interestingly, scaffold disruption prevents this activity-induced ERK and mTOR activation. This observation is coherent with our findings showing that scaffold disruption impairs activation of NMDA receptors [21] (Fig. 2A) which are necessary to induce LTP via Mtor [51].

As discussed previously, our data show subcellular differences in the capacity of mGlu5 and Homer to interact (soma) or not (spines) following a transient neuronal activation in WT mice. Two nonexclusive hypotheses could explain this subcellular difference. First, Shank3 expression is confined to dendritic spines. Hence, counterintuitively, when mGlu5-Homer interaction is broken, Shank3 would be required to keep them apart from one another. Indeed, in the Shank $3 \Delta \mathrm{C}$ mouse model of ASD displaying a truncated form of Shank3, which cannot interact with Homer, mGlu5-Homer interaction dynamics in spines are reminiscent of 
the dynamics in the soma, i.e., a labile interaction highly sensitive to membrane potential and reversible at will. Second, NMDA receptor activation is required for long-lasting disruption of mGlu5-Homer interaction and NMDA/AMPA ratio is decreased in Shank $3 \triangle C$ mice. Thus, a decrease in NMDA currents in Shank $3 \Delta C$ mice could also account for impaired long-lasting disruption of Homer-mGlu5 interaction in spines. These two synergic mechanisms would explain the loss of long-lasting mGlu5-Homer disruption in Shank3 $\Delta C$ mice. In support of this assumption, we show that scaffold disruption in WT mice (using competitive TAT peptides) is sufficient to reduce by half the NMDA/AMPA ratio in CA1 pyramidal cells, corroborating a previous study showing a significant reduction of NMDA currents in similar conditions [35]. Conversely, in Shank3 $\Delta \mathrm{C}$ mice, which display a higher basal endogenous mGlu5-NMDA interaction and lower NMDA/AMPA ratio, repair of the scaffolding complex and its sensitivity to neuronal activity using a chimeric protein is sufficient to restore the NMDA/AMPA ratio.

The chimeric protein also rescues normal synaptic plasticity in Shank3 $\Delta C$ mice. The present study demonstrates that mGlu5 receptosome integrity is required for induction of synaptic plasticity, and suggests the importance of the long-lasting disruption between mGlu5 and Homer for plasticity expression. Arguing in favor of this hypothesis, we previously showed that Homer1a expression was necessary to induce mGlu5-NMDA interaction and blockade of NMDA receptor activity after LTP induction [21]. NMDA blockade prevents further change of synaptic excitability. We can easily speculate that Homer1a interaction with mGlu5 would prevent its reassociation with the multimeric Homer and promote long-lasting disruption of the complex. The long-lasting disruption of the complex following transient depolarization is a common feature of native and restored complexes, enabling plasticity. Further studies will be required to incriminate the long-lasting disruption of the complex in plasticity expression.

In Shank3 $\triangle C$ ASD model, NMDA/AMPA current ratio is decreased compared to WT. The short-lived disruption of mGlu5-Homer interaction induced by transient depolarization is decoupled from the activity of glutamatergic receptors. Consistently, we show that ERK and mTOR signaling are not sensitive to the neuronal activity anymore. Interestingly, we observed an increase of the basal level of activated mTOR in Shank $3 \Delta C$ neurons compared to WT neurons. A saturating activation of mTOR could explain the failure to increase this signaling pathway in Shank3 $\Delta C$ neurons. Overactivation of $\mathrm{mTOR}$ has already been reported in other ASD models [27, 52, 53]. Importantly, expression of the Homer-GluN2B chimaera to restore the integrity of the mGlu5 receptosome in Shank $3 \Delta C$ neurons was sufficient to set mTOR back to its basal level and allow its activation following transient depolarization. Our results show that glutamate receptosome remodeling is necessary to induce NMDA-dependent plasticity and activation of ERK and mTOR signaling pathways.

Autistic patients present two main symptoms: (1) deficits in social communication and (2) stereotyped behaviors meaning restricted and repetitive behavior, interest, or activities. There are also numerous comorbidities such as anxiety, intellectual disability, cognitive deficits, and avoidance behavior to novelty or motor control difficulties. Shank $3 \Delta C$ mice recapitulate the phenotypic features of autistic patients. Among them, and as previously described, we found elevated anxiety, repetitive behaviors, and decreased locomotor performances [7, 8, 10]. The molecular rescue of glutamate receptosome dynamics using Homer-GluN2B chimaera efficiently rescued anxiety-like and repetitive stereotyped behaviors. Expression of anxiety has been associated with an alteration in synaptic plasticity in the limbic circuit [54, 55]. Interestingly, viral infection in both lateral ventricles early during development has allowed a broad expression of the Homer-GluN2B chimaera in both dorsal and ventral hippocampi, as well as other subcortical structures that are part of the limbic system such as striatum and amygdala. Based on our previous results, a deficit in synaptic plasticity caused by the loss of glutamate receptosome dynamics would trigger anxietyrelated behaviors expression in Shank $3 \Delta C$ mice. In contrast, Homer-GluN2B chimaera injection into the lateral ventricles of Shank3 $\Delta C$ mice did not rescue locomotion nor avoidance behavior to novelty. This discrepancy presumably arises from the nature of the brain areas targeted by the injection (Supplementary Fig. S8), and their involvement in specific behaviors. For example, given the cerebellar contribution to locomotor behavior [56] and its relationship with approach/ avoidance traits [57], the absence of transgene expression in the cerebellum is coherent with a lack of recovery of locomotion and avoidance behavior to novelty. Homer-GluN2B chimaera thus seems to be an opportune rescue strategy in the field of ASD. Focusing on the understanding of the molecular dynamics in Shank $3 \Delta C$ mice, we did not test in this study a potential effect of Homer-GluN2B chimaera expressed in WT mice. We might expect a disequilibrium in the molecular dynamics at synapses governing neuronal transmission, plasticity, and cognitive processes, unless proteins from the receptosome that are endogenously expressed are the safeguard of the number of functional receptosomes. Finally, still in a therapeutic perspective, immunohistochemistry performed on brains collected after behavioral experiments showed no sign of morphological disturbances of the cells, suggesting that Homer-GluN2B chimaera is not driving excitotoxicity (Supplementary Fig. S8D). Hence, this therapeutic gene therapy in mice opens promising opportunities to improve cognition in ASD patients by targeting the glutamate receptosome dynamics. In agreement with a recent study performed in adults [58], this work supports promising opportunities to treat neurodevelopmental disorders by rescuing synaptopathies after birth.

In conclusion, in this study, we showed that the reestablishment of glutamate receptosome integrity and dynamics in the Shank $3 \Delta C$ mouse model of ASD, using a chimaera, rescues normal functioning of glutamate receptors and activity-induced neuronal signaling. Importantly, this therapeutic strategy also relieves autistic behaviors in the mice, indicating a causative link between glutamate receptosome alterations and cognitive behaviors in Shankopathies. Other Shankopathies are sensitive to the pharmacological regulation of mGlu5 receptor activity $[41,59]$. ASD in other disorders also relies on mGlu5-Homer interaction dysfunctions, such as fragile $X$ syndrome $[35,42]$ or phenylketonuria, a metabolic disorder that is characterized by autism and intellectual disability if untreated [60]. Therefore, the herein defined therapeutic strategy may be efficiently extended to these related pathologies.

\section{REFERENCES}

1. Bourgeron T. From the genetic architecture to synaptic plasticity in autism spectrum disorder. Nat Neurosci. 2015;16:551-63.

2. Durand CM, Betancur C, Boeckers TM, Bockmann J, Chaste P, Fauchereau F, et al, Mutations in the gene encoding the synaptic scaffolding protein SHANK3 are associated with autism spectrum disorders. Nat Genet. 2007;39:25-7.

3. Carbonetto S. A blueprint for research on shankopathies: a view from research on autism spectrum disorder. Dev Neurobiol. 2013;74:85-112.

4. Betancur C, Buxbaum JD. SHANK3 haploinsufficiency: a "common" but underdiagnosed highly penetrant monogenic cause of autism spectrum disorders. Mol Autism. 2013;4:1.

5. Leblond CS, Nava C, Polge A, Gauthier J, Huguet G, Lumbroso S, et al. Metaanalysis of SHANK mutations in autism spectrum disorders: a gradient of severity in cognitive impairments. PLoS Genet. 2014;10:e1004580.

6. Duffney $\sqcup$, Wei J, Cheng J, Liu W, Smith KR, Kittler JT, et al. Shank3 deficiency induces NMDA receptor hypofunction via an actin-dependent mechanism. J Neurosci. 2013;33:15767-78. http://www.jneurosci.org/cgi/doi/10.1523/JNEUROSCI.1175 13.2013. 
7. Kouser M, Speed HE, Dewey CM, Reimers JM, Widman AJ, Gupta N, et al. Loss of predominant Shank3 isoforms results in hippocampus-dependent impairments in behavior and synaptic transmission. J Neurosci. 2013;33:18448-68. http://www. pubmedcentral.nih.gov/articlerender.fcgi?artid $=3834052 \&$ tool $=$ pmcentrez\& rendertype $=$ abstract.

8. Duffney $\mathrm{L}$, Zhong $\mathrm{P}$, Wei J, Matas E, Cheng J, Qin L, et al. Autism-like deficits in Shank3-deficient mice are rescued by targeting actin regulators. Cell Rep. 2015;11:1400-13.

9. Sala C, Vicidomini C, Bigi I, Mossa A, Verpelli C. Shank synaptic scaffold proteins: keys to understanding the pathogenesis of autism and other synaptic disorders. J Neurochem. 2015;135:849-58.

10. Qinin L, Ma K, Wang ZJ, Hu Z, Matas E, Wei J, et al. Social deficits in Shank3deficient mouse models of autism are rescued by histone deacetylase (HDAC) inhibition. Nat Neurosci. 2018;21:564-75.

11. Bonaglia MC, Giorda R, Borgatti R, Felisari G, Gagliardi C, Selicorni A, et al. Disruption of the ProSAP2 gene in a $t(12 ; 22)(q 24.1 ; q 13.3)$ is associated with the 22q13.3 deletion syndrome. Am J Hum Genet. 2001;69:261-8.

12. Sheng M, Kim E. The postsynaptic organization of synapses. Cold Spring Harb Perspect Biol. 2011;3:a005678.

13. Naisbitt S, Kim E, Tu JC, Xiao B, Sala C, Valtschanoff J, et al. Shank, a novel family of postsynaptic density proteins that binds to the NMDA receptor/PSD-95/GKAP complex and cortactin. Neuron. 1999;23:569-82.

14. Tu JC, Xiao B, Naisbitt S, Yuan JP, Petralia RS, Brakeman P, et al. Coupling of mGluR/Homer and PSD-95 complexes by the Shank family of postsynaptic density proteins. Neuron. 1999;23:583-92.

15. Boeckers TM, Bockmann J, Kreutz MR, Gundelfinger ED. ProSAP/Shank proteinsa family of higher order organizing molecules of the postsynaptic density with an emerging role in human neurological disease. J Neurochem. 2002;81:903-10.

16. Hayashi MK, Tang C, Verpelli C, Narayanan R, Stearns MH, Xu R, et al. The postsynaptic density proteins Homer and Shank form a polymeric network structure. Cell. 2009;137:159-71.

17. Monteiro P, Feng G. SHANK proteins: roles at the synapse and in autism spectrum disorder. Nat Rev Neurosci. 2017;18:147-57.

18. Bruneau EG, Esteban JA, Akaaboune M. Receptor-associated proteins and synaptic plasticity. FASEB J. 2009;23:679-88.

19. Scheefhals N, MacGillavry HD. Functional organization of postsynaptic glutamate receptors. Mol Cell Neurosci. 2018;91:82-94.

20. Christopher M. Synaptic activity induces input-specific rearrangements in a targeted synaptic protein interaction network. Physiol Behav. 2016;176:100-6.

21. Moutin E, Raynaud F, Roger J, Pellegrino E, Homburger V, Bertaso F, et al. Dynamic remodeling of scaffold interactions in dendritic spines controls synaptic excitability. J Cell Biol. 2012;198:251-63.

22. Xiao B, Tu JC, Petralia RS, Yuan JP, Doan A, Breder CD, et al. Homer regulates the association of group 1 metabotropic glutamate receptors with multivalent complexes of Homer-related, synaptic proteins. Neuron. 1998;21:707-16.

23. Beneken J, Tu JC, Xiao B, Nuriya M, Yuan JP, Worley PF, et al. Structure of the Homer EVH1 domain-peptide complex reveals a new twist in polyproline recognition. Neuron. 2000;26:143-54.

24. Hayashi MK, Ames HM, Hayashi Y. Tetrameric hub structure of postsynaptic scaffolding protein Homer. J Neurosci. 2006;26:8492-501.

25. Perroy J, Raynaud F, Homburger V, Rousset M-C, Telley L, Bockaert J, et al. Direct interaction enables cross-talk between ionotropic and group I metabotropic glutamate receptors. J Biol Chem. 2008;283:6799-805. http://www.ncbi.nlm.nih. gov/pubmed/18182392.

26. Goyet E, Bouquier N, Ollendorff V, Perroy J. Fast and high resolution single-cell BRET imaging. Sci Rep. 2016;6:1-12.

27. Bouquierouquier N, Moutin E, Tintignac LA, Reverbel A, Jublanc E, Sinnreich M, et al. AIMTOR, a BRET biosensor for live imaging, reveals subcellular mTOR signaling and dysfunctions. BMC Biol. 2020;18:1-17.

28. Ronesi JA, Collins KA, Hays SA, Tsai NP, Guo W, Birnbaum SG, et al. Disrupted Homer scaffolds mediate abnormal mGluR5 function in a mouse model of fragile $X$ syndrome. Nat Neurosci. 2012;15:431-40.

29. Moutin E, Hemonnot A-L, Seube V, Linck N, Rassendren F, Perroy J, et al. Procedures for culturing and genetically manipulating murine hippocampal postnatal neurons. Front Synaptic Neurosci. 2020;12:19.

30. Coulon V, Audet M, Homburger V, Bockaert J, Fagni L, Bouvier M, et al. Subcellular imaging of dynamic protein interactions by bioluminescence resonance energy transfer. Biophys J. 2008;94:1001-9.

31. Chastagnier $Y$, Moutin E, Hemonnot A, Perroy J. Image processing for bioluminescence resonance energy transfer measurement-BRET-analyzer. Front Comput Neurosci. 2018;11:1-8.

32. De Bundel D, Zussy C, Espallergues J, Gerfen CR, Girault JA, Valjent E. Dopamine D2 receptors gate generalization of conditioned threat responses through mTORC1 signaling in the extended amygdala. Mol Psychiatry. 2016;21:1545-53.
33. Bading H, Ginty DD, Greenberg ME. Regulation of gene expression in hippocampal neurons by distinct calcium signaling pathways. Science. 1993;260:181-6.

34. Sergé A, Fourgeaud L, Hémar A, Choquet D. Receptor activation and homer differentially control the lateral mobility of metabotropic glutamate receptor 5 in the neuronal membrane. J Neurosci. 2002;22:3910-20.

35. Aloisi E, Corf K Le, Dupuis J, Zhang P, Ginger M, Labrousse V, et al. Altered surface mGluR5 dynamics provoke synaptic NMDAR dysfunction and cognitive defects in Fmr1 knockout mice. Nat Commun. 2017. https://doi.org/10.1038/s41467-01701191-2.

36. Araki $Y$, Zeng $M$, Zhang $M$, Huganir RL. Rapid dispersion of SynGAP from synaptic spines triggers AMPA receptor insertion and spine enlargement during LTP. Neuron. 2015;85:173-89.

37. Drapeau E, Riad M, Kajiwara Y, Buxbaum JD. Behavioral phenotyping of an improved mouse model of Phelan-McDermid syndrome with a complete deletion of the Shank3 gene. eNeuro 2018;5:ENEURO.0046-18.

38. Yang M, Bozdagi O, Scattoni ML, Wöhr M, Roullet Fl, Katz AM, et al. Reduced excitatory neurotransmission and mild Autism-Relevant phenotypes in adolescent shank3 null mutant mice. J Neurosci. 2012;32:6525-41.

39. Jaramillo TC, Speed HE, Xuan Z, Reimers JM, Liu S, Powell CM. Altered striatal synaptic function and abnormal behaviour in Shank3 Exon4-9 deletion mouse model of autism. Autism Res. 2016;9:350-75.

40. Drapeau E, Dorr NP, Elder GA, Buxbaum JD. Absence of strong strain effects in behavioral analyses of Shank3-deficient mice. Dis Model Mech. 2014;7:667-81.

41. Wang X, Bey AL, Katz BM, Badea A, Kim N, David LK, et al. Altered mGluR5-Homer scaffolds and corticostriatal connectivity in a Shank3 complete knockout model of autism. Nat Commun. 2016;7:1-18.

42. Guo W, Ceolin L, Collins KA, Perroy J, Huber KM. Elevated CaMKIla and hyperphosphorylation of homer mediate circuit dysfunction in a fragile $X$ syndrome mouse model. Cell Rep. 2015;13:2297-311. http://www.pubmedcentral.nih.gov/ articlerender.fcgi?artid $=4685008 \&$ tool $=$ pmcentrez\&rendertype $=$ abstract.

43. Chokshi V, Gao M, Grier BD, Owens A, Wang H, Worley PF, et al. Input-specific metaplasticity in the visual cortex requires Homer1a-mediated mGluR5 signaling. Neuron. 2019;104:736-48.e6.

44. Hu J-H, Park JM, Park S, Xiao B, Dehoff MH, Kim S, et al. Homeostatic scaling requires group I mGluR activation mediated by Homer1a. Neuron. 2010;68:1128-42.

45. Diering GH, Nirujogi RS, Roth RH, Worley PF, Pandey A, Huganir RL. Homer1a drives homeostatic scaling-down of excitatory synapses during sleep. Science. 2017;515:511-5.

46. Vazdarjanova A, McNaughton BL, Barnes CA, Worley PF, Guzowski JF. Experiencedependent coincident expression of the effector immediate-early genes Arc and Homer 1a in hippocampal and neocortical neuronal networks. J Neurosci. 2002;22:10067-71.

47. English JD, Sweatt JD. A requirement for the mitogen-activated protein kinase cascade in hippocampal long term potentiation. J Biol Chem. 1997;272:19103-6.

48. Schafe GE, Atkins CM, Swank MW, Bauer EP, Sweatt JD, Ledoux JE. Activation of ERK/MAP kinase in the amygdala is required for memory consolidation of Pavlovian fear conditioning. J Neurosci. 2000;20:8177-87.

49. Bockaert J, Marin P. mTOR in brain physiology and pathologies. Physiol Rev. 2015;95:1157-87.

50. Hoeffer CA, Klann E. mTOR signaling: at the crossroads of plasticity, memory and disease. Trends Neurosci. 2010;33:67-75.

51. Cammalleri M, Lütjens R, Berton F, King AR, Simpson C, Francesconi W, et al. Time-restricted role for dendritic activation of the mTOR-p70 S6K pathway in the induction of late-phase long-term potentiation in the CA1. Proc Natl Acad Sci USA. 2003;100:14368-73.

52. Huber KM, Klann E, Costa-Mattioli M, Zukin RS. Dysregulation of mammalian target of rapamycin signaling in mouse models of autism. J Neurosci. 2015;35:13836-42.

53. Sharma A, Hoeffer CA, Takayasu Y, Miyawaki T, McBride SM, Klann E, et al. Dysregulation of mTOR signaling in fragile $X$ syndrome. J Neurosci. 2010;30:694-702.

54. Leuner B, Shors TJ. Stress, anxiety, and dendritic spines: what are the connections? Neuroscience. 2013;251:108-19.

55. Mennesson M, Orav E, Gigliotta A, Kulesskaya N, Saarnio S, Kirjavainen A, et al. Kainate receptor auxiliary subunit NETO2-related cued fear conditioning impairments associate with defects in amygdala development and excitability. eNeuro. 2020;7:ENEURO.0541-19.2020.

56. Sathyanesan A, Gallo V. Cerebellar contribution to locomotor behavior: a neurodevelopmental perspective. Neurobiol Learn Mem. 2019;165:106861.

57. Petrosini L, Cutuli D, Picerni E, Laricchiuta D. Viewing the personality traits through a cerebellar lens: a focus on the constructs of novelty seeking, harm avoidance, and alexithymia. Cerebellum. 2017;16:178-90.

58. Mei Y, Monteiro P, Zhou Y, Kim J-A, Gao X, Fu Z, et al. Adult restoration of Shank3 expression rescues selective autistic-like phenotypes. Nature. 2016;530:481-4. 
59. Vicidomini C, Ponzoni L, Lim D, Schmeisser MJ, Reim D, Morello N, et al. Pharmacological enhancement of mGlu5 receptors rescues behavioral deficits in SHANK3 knock-out mice. Mol Psychiatry. 2016:1-14. https://doi.org/10.1038/ mp.2016.30.

60. Nardecchia F, Orlando R, lacovelli L, Colamartino M, Fiori E, Leuzzi V, et al. Targeting mGlu5 metabotropic glutamate receptors in the treatment of cognitive dysfunction in a mouse model of phenylketonuria. Front Neurosci. 2018;12:1-12.

\section{ACKNOWLEDGEMENTS}

The authors thank the iExplore animal facility (IGF, Montpellier), CompAn behavioral phenotyping facility (MMDN, Montpellier), and the Arpege platform (IGF, Montpellier) for the use of the Infinite F500 plate reader for cell-population BRET. The authors thank Muriel Asari for illustrations. The authors thank Hélène Hirbec for helping with statistical analysis and Giorgio Cignitti for helping with patch on slices. The authors also gratefully acknowledge Paul F. Worley for the generous gift of the Shank $3 \Delta C$ mouse model. The authors truly thank Dr. Amanda Hernan for proofreading. This work was supported by the Agence Nationale de la Recherche (VC, ANR-18-CE160011-01) and the European Research Council (ERC) under the European Union's Horizon 2020 research and innovation programme (JP, grant agreement No. 646788), ANR Lanthslider (JP, ANR-17-CE11-0046), Comitato Telethon Fondazione Onlus (grant no. GGP16131 to CV and GGP17176 to CS), and Regione Lombardia NeOn Progetto “NeOn" POR-FESR 2014-2020, ID 239047, CUP E47F17000000009 to CS and CV).

\section{AUTHOR CONTRIBUTIONS}

EM and JP conceived research and wrote the manuscript; JP, EM, VC, CV, CS, TM, EA, LG, NBo, and SS designed research experiments; VC, NBo, and FG cloned all plasmids.
VC and EM produced viruses. EM performed cell-population BRET experiments; EM, JP, VS, and A-LH-G performed microscopy BRET experiments. EG performed single particle training experiments. NBe analyzed single particle training experiments, with the help of EG and LG. Author EM performed electrophysiology in slices, with the help of EA and FR. Author VC performed coimmunoprecipitations with the help of EM and NBo. Authors CV, CS, and FG designed and characterized the Homer-GluN2B construct. EM performed synaptosomes preparations. NBo and EM performed immunohistochemistry on injected brains. SS performed surgery and viral injections. SS, BG, and JA performed behavioral experiments with the help of TM. Author YC developed toolsets for BRET analysis. JP supervised the project. All authors contributed to the preparation of the manuscript and approved it.

\section{COMPETING INTERESTS}

The authors declare no competing interests. 